\title{
Article
}

Mycosphere

\section{One hundred and five species of lichenicolous biota from India: An updated checklist for the country}

\section{Joshi $\mathbf{Y}^{1,2^{*}}$, Falswal $\mathrm{A}^{2}$, Tripathi $\mathrm{M}^{2}$, Upadhyay $\mathrm{S}^{2,3}$, Bisht $\mathrm{A}^{2}$, Chandra $\mathrm{K}^{2}$, Bajpai $\mathbf{R}^{4}$ and Upreti DK $^{4}$}

\author{
${ }^{1}$ Department of Forest Botany, Kerala Forest Research Institute, Peechi-680653, Kerala, India \\ ${ }^{2}$ Lichenology laboratory, Department of Botany, S.S.J. Campus, Kumaun University, Almora - 263601, Uttarakhand, \\ India \\ ${ }^{3}$ G.B. Pant Institute of Himalayan Environment and Development, Kosi-Katarmal, Almora - 263643, Uttarakhand, \\ India \\ ${ }^{4}$ Lichenology Division, National Botanical Research Institute, Rana Pratap Marg, Lucknow - 226001, Uttar Pradesh, \\ India
}

Joshi Y, Falswal A, Tripathi M, Upadhyay S, Bisht A, Chandra K, Bajpai R, Upreti DK 2016 One hundred and five species of lichenicolous biota from India: An updated checklist for the country. Mycosphere 7(3), 268-294, Doi 10.5943/mycosphere/7/3/3

\begin{abstract}
The knowledge about lichenicolous fungi and lichenicolous lichens occurring in India is summarized. Data on altogether 105 taxa are presented of which 51 species viz. Abrothallus parmeliarum, Acremonium lichenicola, Arthonia clemens, A. epiphyscia, A. phaeophysciae, A. subconveniens, Bellemerella acarosporae, Briancoppinsia cytospora, Buelliella lecanorae, B. minimula, B. protoparmeliopseos, Caeruleoconidia ochrolechiae, Carbonea aggregantula, $C$. assimilis, Cercidospora caudata, C. werneri, Dactylospora homoclinella, D. saxatilis, Didymocyrtis ramalinae, Endococcus propinquus, E. perpusillus, Geltingia associata, Intralichen lichenicola, Kalchbrenneriella cyanescens, Labrocarpon canariensis, Lichenochora verrucicola, Lichenoconium lecanorae, Lichenostigma maureri, L. triseptatum, Lichenothelia convexa, Monerolechia badia, Muellerella lichenicola, M. ventosicola, Odontotrema pertusariae, Opegrapha brigantina, Polycoccum microsticticum, P. peltigerae, Polysporina subfuscescens, Rhymbocarpus pertusariae, Sclerococcum simplex, S. sphaerale, Sphaerellothecium atryneae, S. contextum, S. propinquellum, S. reticulatum, Spirographa fusisporella, Stigmidium cerinae, S. frigidum, S. xanthoparmeliarum, Taeniolella delicata and Zwackhiomyces lecanorae are recorded for the first time from India. Of these 105 species, 103 species are lichenicolous fungi and two species of lichens occur on other lichens, which makes the country one of the best-studied areas in Asia regarding lichenicolous mycobiota. Nine species of lichenicolous fungi or lichens are reported from new host genera. Dendriscocaulon umhausense, reported from Kerala and Tamil Nadu, is excluded from the study, since it is not lichenicolous but forms a photomorph.
\end{abstract}

Key words - Anamorphic fungi - Asia - Ascomycetes - Basidiomycetes - Biodiversity Coelomycetes - Hyphomycetes 


\section{Introduction}

As compared to other countries, knowledge of the presence and distribution of lichenicolous fungi and lichenicolous lichens in India is still rather poor. The lichenicolous biota of India has only recently started to be studied in detail and remains scarcely known. So far, 52 species of lichenicolous fungi and one lichenicolous lichen species have been reported from India (Singh \& Sinha 2010, Joshi 2015a,b). Subsequently to a previous compilation of the information on lichenicolous biota in India, which recognized 52 species (Joshi et al. 2015a,b), here we are reporting further lichenicolous species of India, of which 51 are new to India. Of a total of 105 species of lichenicolous taxa now known from India, 103 are lichenicolous fungi and two are lichenicolous lichens. The highest richness of lichenicolous fungi was recorded from Jammu and Kashmir (53 species), followed by Uttarakhand (50), Himachal Pradesh (20), Madhya Pradesh (9), Sikkim (8), Tamil Nadu (7), Kerala (4), Assam (2) and Arunachal Pradesh, Karnataka, Manipur, Rajasthan, West Bengal (1 each).

This checklist adds some new records of lichenicolous biota to India and establishes a baseline for further studies on this diverse guild of fungi. We predict that the actual richness of the lichenicolous biota will be substantially higher than current estimates and many more discoveries are to be expected. Due to the small number of collections and collectors of lichenicolous fungi in India, we have no clear idea of the distribution of these species in India or the continent or world as a whole.

Our present work is largely a compilation of information present in other workers' publications as well as our own collections. Despite this, we found it useful to produce a comprehensive checklist of these fungi, as we believe that one of the main reasons for the comparatively scant attention these organisms have received is the lack of such checklists, making lichenologists and mycologists unaware of their occurrence in India. For instance, Singh \& Sinha's (2010) compendium of Indian lichens covers a broad taxonomic spectrum, including ca. 2305 species, but only reports the occurrence of two species of lichenicolous fungi [Carbonea vitellinaria (Nyl.) Hertel and Polysporina dubia (Magn.) Vězda].

The aims of this study thus were: to produce an annotated checklist of the lichenicolous biota currently known from India and present new data on the distribution of the 105 species listed. The available data show a fragmentary and unbalanced knowledge of these organisms in India, and the main objective of this paper is to mitigate this situation. We are aware that any species checklist, including distribution assessments, in an area will only hold for a short time. Continued taxonomic investigations and more detailed fieldwork will undoubtedly generate many further additions and modifications to this list.

\section{Materials \& Methods}

The checklist is based on the vouchers deposited at the herbaria of Kerala Forest Research Institute (KFRI) and CSIR-National Botanical Research Institute (LWG) including the personal herbarium of D. D. Awasthi (AWAS) and Lucknow University (LWU) as well as reports and determinations in the literature by earlier workers such as Moreau (1951a,b), Awasthi \& Singh (1975), Sherwood et al. (1981), Pant \& Awasthi (1989), Triebel (1989), Awasthi (1991), Hariharan et al. (1996), Coppins \& Kondratyuk (1998), Alstrup \& Ahti (2007), Joshi et al. (2013), Joseph \& Sinha (2015) and Zhurbenko (2013), even in a few cases where the actual specimen is not documented. The materials were examined under stereozoom dissecting microscope (SZM-2LED OPTIKA). Thin hand-cut sections were made for studying the anatomy of fruiting bodies and examined under a compound microscope (B-150DB OPTIKA). Microscopical examination was done in water, $10 \% \mathrm{KOH}(\mathrm{K})$, lactophenol cotton blue (LCB), Melzer's reagent (MLZ), Lugol's iodine, directly (I) or after a $\mathrm{KOH}$ pre-treatment (K/I), Congo red (CR) solution. 
Authors of host lichens are omitted and for each species, a reference is given for a description of the lichenicolous fungus or lichenicolous lichen treated. All the genera and species within genera reported so far are arranged alphabetically. Each taxon is provided with currently accepted name according to the present concept with author(s) name(s), followed by citation and additional references for taxon reported first time from India. Reported hosts as far as available for individual species are provided as an additional information as these are often useful to establish the correct identity of a taxon. The new records are marked with an asterisk (*).

\section{Results}

ABROTHALLUS De Not., Accad. Sci. Ven.-Trent.-Istr.: 1 (1845)

*A. parmeliarum (Sommerf.) Nyl., Bulletin de la Société Linnéenne de Normandie 3: 12 (1869)

Host - Parmelia squarrosa

Known distribution - Sikkim.

Material examined - India, Sikkim, North Sikkim, Kalep before Thangu, alt. 3900 m, on thallus of P. squarrosa colonizing exposed Cedrus deodara tree trunk, 12 Aug 2004, D.K. Upreti, S. Chatterjee \& P.K. Divakar 04-003845 (LWG 20623).

A. peyritschii (Stein) Kotte, Zentbl. Bakt. ParasitKde 24: 76 (1909)

Host - Vulpicida pinastri

Known distribution - Himachal Pradesh.

Notes - The species was reported by Alstrup \& Ahti (2007) from Himachal Pradesh.

ACREMONIUM Link, Magazin der Gesellschaft Naturforschenden Freunde Berlin 3(1): 15 (1809)

*A. lichenicola W. Gams, Cephalosporium-artige Schimmelpilze: 134 (1971)

Host - Usnea vegae

Known distribution - Kerala.

Material examined: India, Kerala, Palakkad district, Silent Valley National Park, Anginda, Cheriya, alt. 2100 m, on U. vegae, s.d., Stephen 23591 (KFRI).

ARTHONIA Ach., Neues Journal für die Botanik 1: 3 (1806)

*A. clemens (Tul.) Th. Fr., Kongliga Svenska Vetenskapsakademiens Handlinger 7: 46 (1867)

Hosts - Rhizoplaca chrysoleuca, R. peltata

Known distribution - Himachal Pradesh, Jammu \& Kashmir, Uttarakhand.

Materials examined: India, Himachal Pradesh, Kinnaur district, in and around Yangthang, alt. $3800 \mathrm{~m}$, on R. peltata colonizing rocks, 02 Nov 2003, Upreti, Srivastava \& Prakash 03-002630 (LWG 23396). Jammu \& Kashmir, Anantanag district, Neh Nar Glacier area, alt. 3960 m, on thallus of $R$. chrysoleuca colonizing exposed quartzite rock, s.d., D.N. Bhattacharya s.n. (LWGAWAS 14816, 14817); Doda district, Badharwah, Ramkund, on R. chrysoleuca colonizing rocks, Aug 2010, Chandra Sekhar 10-012569 (LWG 28073). Uttarakhand, Chamoli district, Badrinath, south of temple, near Brahmini village, alt. 3125-3200 m, on $R$. chrysoleuca colonizing boulders, 27 Sept 1976, K. Dange 76.724 (LWG-LWU); Pithoragarh district, Burfu, en route to Milam glacier, alt. $3250 \mathrm{~m}$, on $R$. chrysoleuca colonizing rocks, 19 Oct 2007, Santosh Joshi 07-010532 (LWG 11522); Uttarkashi district, Gomukh area, right bank, $5^{\text {th }}$ moraine, alt. ca. $3840 \mathrm{~m}$, on $R$. chrysoleuca colonizing boulders, 04 Jul 1976, D.D. Awasthi \& S.R. Singh 8475/A (LWG-AWAS 14907). 
A. diorygmae S. Joshi \& Upreti, The Lichenologist 45: 323 (2013)

Host - Diorygma junghuhnii

Known distribution - Tamil Nadu.

Notes - The species was reported by Joshi et al. (2013) from Tamil Nadu.

*A. epiphyscia Nyl., Flora (Regensburg) 58: 361 (1875)

Hosts - Physcia stellaris, Physcia sp.

Known distribution - Jammu \& Kashmir, Uttarakhand.

Materials examined - India, Uttarakhand, Chamoli district, Badrinath, Mana Village, on Physcia sp. growing along with Xanthoria ulophyllodes colonizing exposed rocks, 21 Jun 2005, V. Shukla \& Y. Joshi 05-005959 (LWG); Uttarkashi district, on way to Gomukh, 6 km from Gangotri, alt. $11000 \mathrm{~m}$, on thallus of P. stellaris colonizing exposed bark, 30 Jun 1976, D.D. Awasthi \& S.R. Singh 8295 (LWG-AWAS 14122). Jammu \& Kashmir, Ladakh, Zanskar, Ichar, on Physcia sp. growing along with Aspicilia maculata colonizing rocks, 08 Jul 2012, Jatinder Kumar 12-019273 (LWG 13355).

A. molendoi (Frauenf.) R. Sant., Thunbergia 3: 2 (1986)

Host - Xanthoria elegans

Known distribution - Himachal Pradesh, Jammu \& Kashmir.

Materials examined - India, Himachal Pradesh, Lahul Spiti district, Keylong, Germaru, alt. $3550 \mathrm{~m}$, on X. elegans colonizing rocks, 14 Sept 2001, D.K. Upreti 01-26540 (LWG); Spiti, Spiti valley, $13 \mathrm{~km}$ before Kiuta from Kaza side, alt. $3800 \mathrm{~m}$, on $X$. elegans colonizing rocks, 06 Aug 2002, Upreti \& Divakar 02-000148 (LWG); Rongrick, alt. 3600 m, on X. elegans colonizing rocks, 04 Aug 2002, Upreti \& Divakar 02-000109 (LWG); Lossar, alt. 3900 m, on X. elegans colonizing rocks, 04 Aug 2002, Upreti \& Divakar 02-00094 (LWG); Zinzibar, alt. $4200 \mathrm{~m}$, on X. elegans colonizing rocks, 04 Aug 2003, Upreti \& Chatterjee 03-001751 (LWG). Jammu \& Kashmir, Ladakh, Zanskar, Rangdeem, on X. elegans colonizing rocks, 11 Sept 2012, Jatinder Kumar 12019325 (LWG 13260); Hemis National Park, Rumbak valley, Spandin, alt. 5000 m, on X. elegans colonizing rocks, 28 Jul 1999, H.R. Negi L26B (LWG).

Notes - The species was previously reported from Jammu \& Kashmir by Zhurbenko (2013) and in the present study; it is extending its distributional range up to Himachal Pradesh.

*A. phaeophysciae Grube \& Matzer, Progress and Problems in Lichenology in the Nineties. Proceedings of the Third Symposium of the International Association for Lichenology 68: 10 (1997)

Host - Phaeophyscia sp.

Known distribution - Uttarakhand.

Material examined: India, Uttarakhand, Almora district, en route to Daniya, Dhauladevi forest, on thallus of Phaeophyscia sp., 16 May 2015, Krishna Chandra \& Shashi Upadhyay s.n. $(\mathrm{KU})$.

*A. subconveniens Nyl., Flora (Regensburg) 50: 440 (1867)

Host - Lobaria meridionalis

Known distribution - Himachal Pradesh.

Material examined: India, Himachal Pradesh, Great Himalayan National Park, Apgain Thack, alt. 2840 m, on L. meridionalis colonizing soil, 10 Jun 1999, D.K. Upreti L65101 (LWG).

BELLEMERELLA Nav.-Ros. \& Cl. Roux, Mycotaxon 61: 443 (1997)

*B. acarosporae Calat. \& Nav.-Ros., Nova Hedwigia 72: 475 (2001)

Host-Acarospora fusca

Known distribution - Uttarakhand. 
Materials examined - India, Uttarakhand, Chamoli district, way to Bhuna, alt. $3750 \mathrm{~m}$, on A. fusca colonizing rocks, 23 Oct 1967, A. Singh \& party 91589 (LWG 000137); on way from Chopta to Tungnath peak, alt. 3600-4200 m, on A. fusca colonizing exposed boulders, 24 Sept 1976, K. Dange 76.668 (LWG).

BIATOROPSIS Räsänen, Annales Botanici Societatis Zoologicae Botanicae Fennicae "Vanamo" 5: 8 (1934)

B. usnearum Räsänen, Annales Botanici Societatis Zoologicae Botanicae Fennicae "Vanamo" 5: 8 (1934)

Host - Usnea austroindica

Known distribution - Tamil Nadu.

Material examined - India, Tamil Nadu, Kanya Kumari, Upper Kodayar, alt. 1050 m, on thallus of corticolous $U$. austroindica, May 2011, Ravichandran s.n. (LWG).

BRIANCOPPINSIA Diederich, Ertz, Lawrey \& van den Boom, Fungal Diversity 52 (1): 7 (2011)

*B. cytospora (Vouaux) Diederich, Ertz, Lawrey \& van den Boom, Fungal Diversity 52 (1): 8 (2011)

Host - Xanthoparmelia mexicana

Known distribution - Sikkim.

Material examined - India, Sikkim, North Sikkim, Thangu area, alt. $4000 \mathrm{~m}$, on $X$. mexicana colonizing rocks, 12 Aug 2004, D.K. Upreti, S. Chatterjee \& P.K. Divakar 04-003896 (LWG).

BUELLIELLA Fink, The lichen flora of the United States: 372 (1935)

*B. lecanorae Suija \& Alstrup, The Lichenologist 36 (3-4): 203 (2004)

Host - Lecanora cf. leproplaca

Known distribution - Uttarakhand.

Material examined - India, Uttarakhand, Dehradun district, Kansar, Forest Guest House, alt. 2400 m, on L. cf. leproplaca, 13 Aug 2001, D.K. Upreti 01-78372 (LWG 15756).

*B. minimula (Tuck.) Fink, The lichen flora of the United States: 372 (1935)

Hosts - Ochrolechia rosella, Pertusaria leucostoma, Pertusaria sp.

Known distribution - Madhya Pradesh, Uttarakhand.

Materials examined - India, Madhya Pradesh, Anoopur district, Amarkantak, Mai ki Bagia, alt. 612 m, on P. leucostoma colonizing bark of Shorea robusta, 23 Mar 2004, Upreti, Nayaka \& Satya 04-002439 (LWG 009888); Dindori district, Tarwartola near to Chauradader, alt. $1480 \mathrm{~m}$, on P. leucostoma colonizing bark of S. robusta, 06 Jul 2005, Upreti, Nayaka \& Satya 05-005692/A (LWG 009884). Uttarakhand, Pithoragarh district, Munsyari, Khaliya top, alt. 2700-3000 m, on $O$. rosella colonizing bark, 31 Oct 2009, D.K. Upreti \& party 09-013462 (LWG 13523).

Notes $-O$. rosella is the new host for this fungus.

*B. protoparmeliopseos Etayo \& Pérez-Ortega, The Lichenologist 42 (3): 273 (2010)

Host - Protoparmeliopsis muralis

Known distribution - Jammu \& Kashmir, Uttarakhand.

Materials examined - India, Jammu \& Kashmir, Gulmarg, on way from Gulmarg to Khilanmarg, alt. 2560-2650 m, on P. muralis colonizing boulders, 03 Jul 1977, K. Dange 77.522 (LWG); Srinagar district, Harwan, Dachigam National Park, alt. 2185 m, on P. muralis colonizing rocks, 02 Aug 2005, M.A. Sheikh 05-006188 (LWG 5484). Uttarakhand, Chamoli district, Malari, alt. 2400 m, on P. muralis colonizing rocks, 24 Sept 2006, S. Rawat 06-006766 (LWG 23420). 
CAERULEOCONIDIA Zhurb. \& Diederich, Herzogia 28: 764 (2015)

*C. ochrolechiae Zhurb. \& Diederich, Herzogia 28: 764 (2015)

Host - Ochrolechia rosella

Known distribution - Uttarakhand.

Material examined - India, Uttarakhand, Almora district, en route to Sunderdhunga Glacier, between Loharkhet and Dhakuri, before $5 \mathrm{~km}$ of Dhakuri, alt. $9000 \mathrm{ft}$, on thallus of O. rosella colonizing exposed Quercus bark, 11 Sept 1995, D.K. Upreti \& Jyoti Tandon 213418 (LWG 13522).

CARBONEA (Hertel) Hertel, Mitteilungen aus der Botanischen Staatssammlung München 19: 441 (1983)

*C. aggregantula (Müll. Arg.) Diederich \& Triebel, Herzogia 16: 51 (2003)

Hosts - Lecanora impudens, L. leproplaca, L. subgranulata, Protoparmeliopsis muralis

Known distribution - Jammu \& Kashmir, Madhya Pradesh, Uttarakhand.

Materials examined - India, Jammu \& Kashmir, Pahalgam, on way to Shikargarh via Mamleshwar, alt. ca. $2250 \mathrm{~m}$, on P. muralis colonizing boulders, 27 Jun 1977, K. Dange 77.157 (LWG-LWU). Madhya Pradesh, Shahdol district, Amarkantak, Kabir Chabutra, on L. impudens colonizing bark of S. robusta, 28 Sept 1987, D.K. Upreti 201763 (LWG 15755); ValkoKhurkhuridadar, $8 \mathrm{~km}$ away from Amarkantak town, alt. $1100 \mathrm{~m}$, on thallus of L. leproplaca colonizing exposed bark of tree, 29 Jan 1980, D.K. Upreti \& U.C. Misra 80-478 (LWG-LWU). Uttarakhand, Almora district (now Pithoragarh district), near Berinag forest rest house, alt. $1830 \mathrm{~m}$, on thallus L. subgranulata colonizing exposed bark of Aesculus indica, 27 Sept 1950, D.D. Awasthi 810 (LWG-AWAS 12553).

*C. assimilis (Körb.) Hafellner \& Hertel, Die Flechten Baden-Württembergs. Verbreitungsatlas: 511 (1987)

Host - Xanthoria elegans

Known distribution - Uttarakhand.

Material examined - India, Uttarakhand, Chamoli district, Malari, on X. elegans colonizing rocks, 24 Sept 2006, S. Rawat 06-006778 (LWG).

Notes - It is a lichenicolous lichen.

C. vitellinaria (Nyl.) Hertel, Mitteilungen aus der Botanischen Staatssammlung München 19: 442 (1983)

Host - Candelaria vitellinaria

Known distribution - Himachal Pradesh, Jammu \& Kashmir.

Material examined - India, Himachal Pradesh, Lahul Spiti district, Sissu area, alt. 3000 m, on C. vitellinaria colonizing exposed rocks, 13 Sept 2001, D.K. Upreti 01-26528/A (LWG).

Notes - The species was previously reported from Jammu \& Kashmir by Hertel (1983), Poelt (1961) and Zhurbenko (2013) and in the present study; it is extending its distributional range up to Himachal Pradesh.

CERCIDOSPORA Körb., Parerga lichenologica. Ergänzungen zum Systema lichenum Germaniae: 465 (1865)

*C. caudata Kernst., Verhandlungen der Zoologisch-Botanischen Gesellschaft Wien 44: 212 (1894)

Hosts - Caloplaca approximata, C. biatorina var. biatorina, Xanthoria elegans

Known distribution - Himachal Pradesh, Jammu \& Kashmir, Uttarakhand. 
Materials examined - India, Himachal Pradesh, Lahul Spiti district, Spiti valley, Poh, alt. $3300 \mathrm{~m}$, on C. biatorina var. biatorina colonizing exposed rocks, 05 Aug 2002, Upreti \& Divakar 02-000130 (LWG 008746); Zinzibar, alt. $4200 \mathrm{~m}$, on Xanthoria colonizing exposed rocks, 04 Aug 2003, D.K. Upreti \& S. Chatterjee 03-001761/A (LWG 23391); Patrio, alt. $3800 \mathrm{~m}$, on X. elegans colonizing exposed rocks, 04 Aug 2003, D.K. Upreti \& S. Chatterjee 03-001737 (LWG). Jammu \& Kashmir, Leh district, Leh (Dihar), on X. elegans colonizing exposed rocks, 12 Jun 2010, Jatinder Kumar 10-14304 (LWG); Ladakh, Chunthang valley, Sorinsa, on X. elegans colonizing exposed rocks, 02 Aug 2012, Jatinder Kumar 12-019248 (LWG). Uttarakhand, Bageshwar district, Pindari glacier, near Babaji's hut, on C. approximata colonizing exposed rocks, s.d., Y. Joshi \& S. Joshi 07010315 (LWG); Pithoragarh district, Burfu, en route to Milam glacier, alt. $3250 \mathrm{~m}$, on X. elegans colonizing rocks, 19 Oct 2007, Santosh Joshi 07-010545 (LWG 11733).

C. macrospora (Uloth) Hafellner \& Nav.-Ros., Lichen Flora of the Greater Sonoran Desert Region 2: 638 (2004)

Hosts - Lecanora sp., Protoparmeliopsis muralis var. dubyi, P. muralis var. muralis

Known distribution - Himachal Pradesh, Jammu \& Kashmir.

Materials examined - India, Himachal Pradesh, Lahul Spiti district, Koksar, alt. 27-3000 m, on P. muralis var. dubyi colonizing rocks, 13 Sept 2001, D.K. Upreti 01-26520/D (LWG 15377). Jammu \& Kashmir, Ladakh district, Kargil, on P. muralis colonizing rocks, Aug 1979, A. Singh \& M. Ranjan L18306 (LWG); Shankaracharya hill, alt. ca. $1645 \mathrm{~m}$, on P. muralis var. muralis colonizing rocks, 15 May 1970, P.N. Mujoo 70.88 (LWG-LWU).

Notes - The species was previously reported from Jammu \& Kashmir by Zhurbenko (2013) and in the present study; it is extending its distributional range up to Himachal Pradesh.

C. melanophthalmae Nav.-Ros., Calat. \& Hafellner, Mycosphere 4 (3): 551 (2013)

Hosts - Rhizoplaca chrysoleuca, $R$. melanophthalma var. melanophthalma, $R$. melanophthalma var. obscura, R. peltata

Known distribution - Himachal Pradesh, Jammu \& Kashmir, Uttarakhand.

Materials examined - India, Himachal Pradesh, Kangra district, Taktsi valley, near Spiti river, alt. $3810 \mathrm{~m}$, on $R$. melanophthalma var. melanophthalma colonizing stones and boulders, 11 Aug 1952, O.A. Höeg 1674 (LWG-AWAS 14814); Lahaul Spiti district, Spiti valley, Lossar. alt. $3900 \mathrm{~m}$, on $R$. melanophthalma var. melanophthalma colonizing exposed rocks, 04 Aug 2002, Upreti \& Divakar 02-000093 (LWG 23413); Darcha, alt. $3200 \mathrm{~m}$, on R. melanophthalma colonizing exposed rocks, 04 Aug 2003, D.K. Upreti \& S. Chatterjee 03-001732 (LWG 23409); Zinzibar, alt. $4200 \mathrm{~m}$, on $R$. peltata colonizing exposed rocks, 04 Aug 2003, D.K. Upreti \& S. Chatterjee 03-001761/A (LWG 23391). Jammu \& Kashmir, Ladakh, Sourire, on $R$. chrysoleuca colonizing rocks, 02 Aug 2012, Jatinder Kumar (DIHAR) 12-019252 (LWG 13186); Suru, Panikar, on $R$. peltata colonizing rocks, 12 Sept 2012, Jatinder Kumar 12-019419 (LWG); Leh district, Changla, on $R$. melanophthalma var. obscura colonizing exposed rocks, 05 Jul 2010, Jatinder Kumar 10-014330 (LWG 10294). Uttarakhand, Uttarkashi district, Gangotri, between Bhojwasa to Gomukh, alt. $3452 \mathrm{~m}$, on on $R$. melanophthalma colonizing soil over rocks, 23 Oct 2010, Himanshu Rai \& Pramod Nag 10-10017406 (LWG).

Notes - The species was previously reported from Jammu \& Kashmir by Zhurbenko (2013) and in the present study; it is extending its distributional range up to Himachal Pradesh.

*C. werneri Nav.-Ros., Calat. \& Hafellner, Mycotaxon 110: 19 (2009)

Host - Aspicilia sp.

Known distribution - Jammu \& Kashmir.

Material examined - India, Jammu \& Kashmir, Leh district, Changla, on Aspicilia sp. growing along with Acarospora fusca colonizing rocks, 05 Jul 2010, Jatinder Kumar 10-014321 (LWG 10281). 
C. xanthoriae (Wedd.) R. Sant., Lichens and Lichenicolous Fungi of Sweden and Norway: 57 (1993)

Hosts - Xanthoria elegans, X. sorediata

Known distribution - Himachal Pradesh, Jammu \& Kashmir, Uttarakhand.

Materials examined - India, Himachal Pradesh, Lahul Spiti district, Spiti valley, Poh, alt. 3300 m, on Xanthoria colonizing exposed rocks, 05 Aug 2002, D.K. Upreti \& P.K. Divakar 02000129 (LWG); Sissu, alt. 3100 m, on Xanthoria growing along with Acarospora fusca colonizing rocks, 04 Aug 2003, D.K. Upreti \& S. Chatterjee 03-001703 (LWG 005298). Jammu \& Kashmir, Ladakh, Hemis National Park, Rumbak valley, Spandin, alt. 5000 m, on X. elegans colonizing rocks, 28 Jul 1999, H.R. Negi L26B (LWG). Uttarakhand, Almora district, Martoli plain, near Pindari, alt. 3750 m, on X. sorediata colonizing rocks, 27 May 1972, A. Singh 91982 (LWG).

Notes - The species was previously reported from Jammu \& Kashmir by Zhurbenko (2013) and in the present study; it is extending its distributional range up to Himachal Pradesh and Uttarakhand.

\section{Cercidospora sp.}

Host - Lecanora sp.

Known distribution - Jammu \& Kashmir.

Notes - This species was reported by Zhurbenko (2013) from Jammu \& Kashmir.

CLADOSPORIUM Link, Magazin der Gesellschaft Naturforschenden Freunde Berlin 8: 37 (1816)

C. licheniphilum Heuchert \& U. Braun, Herzogia 19: 12 (2006)

Hosts - Pertusaria sp., Xanthoria candelaria

Known distribution - Jammu \& Kashmir, Uttarakhand.

Material examined - India, Uttarakhand, Nainital district, Kilbury forest, near forest rest house, on thallus of Pertusaria colonizing Quercus bark, 19 Apr 2015, Y. Joshi \& A. Bisht s.n. (KU).

Notes - The species was previously reported by Zhurbenko (2013) from Jammu \& Kashmir colonizing thallus of $X$. candelaria and in the present study; it is extending its distributional range up to Uttarakhand where it is colonizing Pertusaria species.

CORTICIFRAGA D. Hawksw. \& R. Sant., Bibliotheca Lichenologica 38: 123 (1990)

C. peltigerae (Fuckel) D. Hawksw. \& R. Sant., Bibliotheca Lichenologica 38: 129 (1990)

Hosts - Peltigera canina, P. elisabethae, P. ponojensis

Known distribution - Jammu \& Kashmir, Uttarakhand.

Material examined - India, Uttarakhand, Uttarkashi district, on way to Gomukh, $11 \mathrm{~km}$ from Gangotri, alt. $3535 \mathrm{~m}$, on thallus of P. canina colonizing exposed mossy soil, 30 Jun 1976, D.D. Awasthi \& S.R. Singh 8369 (LWG-AWAS 13850).

Notes - The species was previously reported from Jammu \& Kashmir by Zhurbenko (2013) and in the present study; it is extending its distributional range up to Uttarakhand.

DACTYLOSPORA Körb., Systema lichenum Germaniae: 271 (1855)

*D. homoclinella (Nyl.) Hafellner, Beihefte zur Nova Hedwigia 62: 112 (1979)

Hosts - Lecanora impudens, Protoparmeliopsis muralis

Known distribution - Himachal Pradesh, Madhya Pradesh.

Materials examined - India, Himachal Pradesh, Lahul Spiti district, Lahul valley, Koksar, alt. $3100 \mathrm{~m}$, on P. muralis colonizing rocks, 02 Aug 2002, D.K. Upreti 02-000031/B (LWG 15554). Madhya Pradesh, Shahdol district, Amarkantak, Valko-Khurkhuri dadar, $8 \mathrm{~km}$ from 
Amarkantak town, alt. ca. $1100 \mathrm{~m}$, on L. impudens colonizing bark of a tree, 29 Jan 1980, D.K. Upreti \& U.C. Misra 80-487 (LWG 16309).

*D. saxatilis (Schaer.) Hafellner, Beihefte zur Nova Hedwigia 62: 129 (1979)

Hosts - Pertusaria indica, P. leucostoma, P. pertusa, Pertusaria sp.

Known distribution - Jammu \& Kashmir, Madhya Pradesh, Uttarakhand.

Materials examined - India, Jammu \& Kashmir, s.d., Vishal Kumar s.n. (KU). Madhya Pradesh, Katni district, Dhirmar Kheda, on thallus of P. leucostoma colonizing bark of Mangifera indica, 20 Feb 2009, G.K. Mishra \& S. Mohabe 09-009911/B (LWG 10547); Piproel, on thallus of P. leucostoma colonizing bark of M. indica, 21 Feb 2009, G.K. Mishra \& S. Mohabe 09-009954 (LWG 10529); Shahdol district, Amarkantak, Valko-Khurkhuridadar, $08 \mathrm{~km}$ from Amarkantak town, alt. ca. $1100 \mathrm{~m}$, on P. pertusa colonizing bark of tree, 29 Jan 1980, D.K. Upreti \& U.C. Misra 80-497 (LWG-LWU). Uttarakhand, Almora district, Binsar, alt. $1800 \mathrm{~m}$, on P. indica colonizing rocks in moist areas, 10 Sept 1995, D.K. Upreti \& Jyoti Tandon 213340 (LWG 14302).

DIDYMOCYRTIS Vain., Acta Societatis pro Fauna et Flora Fennica 49(2): 221 (1921)

*D. ramalinae (Roberge ex Desm.) Ertz, Diederich \& Hafellner, Fungal Diversity 74: 77 (2015)

Host - Ramalina inflata

Known distribution - Kerala.

Material examined - India, Kerala, Palakkad district, Silent Valley National Park, Anginda, alt. 2200 m, on R. inflata, s.d., Stephen 23640 (KFRI).

ENDOCOCCUS Nyl., Mémoires de la Société Impériale des Sciences Naturelles de Cherbourg 3: $193(1855)$

E. incrassatus Etayo \& Breuss, Österreichische Zeitschrift für Pilzkunde 10: 316 (2001)

Host - Endocarpon pusillum

Known distribution - Jammu \& Kashmir.

Notes - The species was reported from Jammu \& Kashmir by Zhurbenko (2013).

*E. propinquus (Körb.) D. Hawksw., Botaniska Notiser 132: 287 (1979)

Hosts - Popidia crustulata, P. macrocarpa

Known distribution - Himachal Pradesh, Uttarakhand.

Materials examined - India, Himachal Pradesh, Lahul Spiti district, Lahul valley, Rohtang pass area, alt. $3900 \mathrm{~m}$, on P. crustulata growing along with Aspicilia and Rhizocarpon sp. colonizing rocks, 02 Aug 2002, Upreti \& Divakar 02-000021/C (LWG 21215). Uttarakhand, Bageshwar district, Pindari glacier, Zero point, alt. $3881 \mathrm{~m}, 30^{\circ} 15^{\prime} 34.5^{\prime \prime} \mathrm{N} 79^{\circ} 59^{\prime} 55.7^{\prime \prime} \mathrm{E}$, on $P$. macrocarpa colonizing rocks, 07 Oct 2015, R. Bajpai \& M. Tripathi 15-027539 (DUP) (LWG); Chamoli district, way from Chopta to Tungnath peak, alt. 3650-4270 m, on P. macrocarpa colonizing rocks, 24 Sept 1976, K. Dange 76-574 (LWG-LWU 14249).

*E. perpusillus Nyl., Actes de la Société Linnéenne de Bordeaux 21: 439 (1857)

Hosts - Aspicilia sp., Candelariella aurella

Known distribution - Himachal Pradesh.

Materials examined - India, Himachal Pradesh, alt. $3400 \mathrm{~m}$, on C. aurella colonizing exposed rocks, 05 Aug 2002, Upreti \& Divakar 02-000112 (LWG); Lahul Spiti district, Darcha, alt. $3200 \mathrm{~m}$, on Aspicilia sp. colonizing rocks, 04 Aug 2003, D.K. Upreti \& S. Chatterjee 03-001715 (LWG 15371).

Notes - Candelariella aurella is a new host for this fungus. 
E. rugulosus (Leight.) Nyl., Mémoires de la Société Impériale des Sciences Naturelles de Cherbourg 3: 193 (1855)

Hosts - Aspicilia sp., Rhizocarpon disporum

Known distribution - Jammu \& Kashmir.

Notes - The species was reported from Jammu \& Kashmir by Triebel (1989) and Zhurbenko (2013).

GELTINGIA Alstrup \& D. Hawksw., Meddelelser om Grønland Biosciences 31: 33 (1990)

*G. associata (Th. Fr.) Alstrup \& D. Hawksw., Meddelelser om Grønland Biosciences 31: 33 (1990)

Host - Thamnolia vermicularis

Known distribution - Sikkim.

Material examined - India, Sikkim, North Sikkim district, Thangu, Chopta valley, alt. 4193

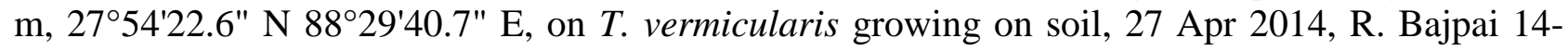
022034 (LWG).

HOMOSTEGIA Fuckel, Jahrbücher des Nassauischen Vereins für Naturkunde 23-24: 223 (1870)

H. hertelii D. Hawksw., V. Atienza \& M.S. Cole, Bibliotheca Lichenologica 88: 189 (2004)

Hosts - Flavoparmelia caperata, Punctelia rudecta

Known distribution - Uttarakhand.

Materials examined - Uttarakhand, Almora district, Dol Ashram, on thallus of P. rudecta colonizing Quercus tree, 15 Mar 2014, M. Tripathi \& party s.n. (KU); Jhakar Saim Forest, on thallus of $F$. caperata and P. rudecta colonizing Quercus tree, 06 Jul 2014, M. Tripathi, K. Chandra \& S. Upadhyay s.n. (KU).

H. piggotii (Berk. \& Broome) P. Karst., Bidrag till Kännedom av Finlands Natur och Folk 23: 222 (1873)

Host - Parmelia sp.

Known distribution - Uttarakhand.

Notes - The species was previously reported from Uttarakhand by Alstrup \& Ahti (2007).

INTRALICHEN D. Hawksw. \& M.S. Cole, Fungal Diversity 11: 88 (2002)

I. christiansenii (D. Hawksw.) D. Hawksw. \& M.S. Cole, Fungal Diversity 11: 90 (2002)

Hosts - Caloplaca approximata, Candelariella aurella, Lobothallia alphoplaca

Known distribution - Jammu \& Kashmir, Uttarakhand.

Materials examined - India, Uttarakhand, Almora district (now Bageshwar district); above Phurkia towards Pindari glacier, alt. $3350 \mathrm{~m}$, on L. alphoplaca colonizing rocks, 23 May 1950, D.D. Awasthi \& A.M. Awasthi 773 (LWG-AWAS 16478); Bageshwar district, Pindari glacier, near Babaji's hut, on C. approximata colonizing exposed rocks, s.d., Y. Joshi \& S. Joshi 07-010315 (LWG).

Notes - The species was previously reported colonizing apothecial disc of Candelariella aurella from Jammu \& Kashmir by Zhurbenko (2013) and in the present study; it is extending its distributional range up to Uttarakhand.

*I. lichenicola (M.S. Christ. \& D. Hawksw.) D. Hawksw. \& M.S. Cole, Fungal Diversity 11: 93 (2002)

Host - Candelariella sp.

Known distribution - Uttarakhand. 
Material examined - India, Uttarakhand, Pithoragarh district, Martoli, en route to Milam glacier, alt. $3390 \mathrm{~m}$, on Candelariella sp. growing along with Xanthoria elegans colonizing rocks, 23 Oct 2007, S. Joshi 07-010540 (LWG).

KALCHBRENNERIELLA Diederich \& M. S. Christ., The Bryologist 105 (3): 411 (2002)

*K. cyanescens (Kalchbr.) Diederich \& M. S. Christ., The Bryologist 105 (3): 411(2002)

Hosts - Usnea fischeri, U. nepalensis, U. orientalis, U. picta, U. rubicunda, $U$. stigmatoides, $U$. subchalybaea

Known distribution - Kerala.

Materials examined - India, Kerala district, Alappuzha district, Punnamada, on Usnea sp., s.d., Stephen 23201 (KFRI); Idukki district, Kanthalloor, Mannavan Shola, alt. ca. 2000 m, on $U$. orientalis colonizing bark of Rhododendron, 13 Jan 1999, Stephen 21990 (KFRI); ibid., alt. ca. $1650 \mathrm{~m}$, on U. stigmatoides colonizing bark, 14 Jan 1999, Stephen 22062 (KFRI); Anaimudi slope, alt. ca. $2300 \mathrm{~m}$, on U. fragilis colonizing bark, 15 Jan 1999, Stephen 22116 (KFRI); Munnar, Lockart Gap, alt. ca. 1600 m, on Usnea sp., 15 Jan 1998, Stephen \& Viswany 008965 (KFRI); Palakkad district, Silent Valley National Park, Anginda, alt. 2200 m, on U. nepalensis, s.d., Stephen 88a, 23649 (KFRI); ibid., on U. orientalis, s.d., Stephen 22149 (KFRI); ibid., on U. picta, s.d., Stephen 23650 (KFRI); ibid., on U. rubicunda, s.d., Stephen 23652 (KFRI); ibid., on $U$. subchalybaea, s.d., Stephen 22150 (KFRI); Sispara, alt. 2050 m, on U. fischeri, s.d., Stephen 81e (KFRI).

LABROCARPON Etayo \& Pérez-Ortega, The Lichenologist 42 (3): 271 (2010)

*L. canariensis (D. Hawksw.) Etayo \& Pérez-Ortega, The Lichenologist 42 (3): 271 (2010)

Host - Pertusaria leucostoma

Known distribution - Madhya Pradesh.

Material examined - India, Madhya Pradesh, Dindori district, around Jagatpur forest rest house, alt. $1300 \mathrm{~m}$, on P. leucostoma colonizing bark of Shorea robusta, 05 Jul 2005, Upreti, Nayaka \& Satya 05-005595 (LWG 009882).

LICHENOCHORA Hafellner, Nova Hedwigia 48 (3-4): 358 (1989)

*L. verrucicola (Wedd.) Nik. Hoffm. \& Hafellner, Bibliotheca Lichenologica 77: 55 (2000)

Host - Aspicilia sp.

Known distribution - Jammu \& Kashmir.

Material examined - India, Jammu \& Kashmir, Leh district, Leh, alt. ca. $3550 \mathrm{~m}$, on Aspicilia colonizing rocks, 12 Jun 2010, Jatinder Kumar 10-014306 (LWG).

LICHENOCONIUM Petr. \& Syd., Feddes Repertorium Specierum Novarum Regni Vegetabilis Beihefte 42: 432

*L. lecanorae (Jaap) D. Hawksw., Bulletin of the British Museum for Natural History 6 (3): 270 (1979)

Hosts - Protoparmeliopsis muralis, Psora sp., Rhizoplaca chrysoleuca

Known distribution - Himachal Pradesh, Uttarakhand.

Materials examined - India, Himachal Pradesh, Kullu district, Great Himalayan National Park, Jiwanala valley, Dwara, alt. ca. 3150 m, on R. chrysoleuca colonizing rocks, 20 Jun 2004, R. Srivastava 04-003647 (LWG 23371); Lahul Spiti district, Darcha, alt. $3200 \mathrm{~m}$, on Psora sp. colonizing rocks, 04 Aug 2003, D.K. Upreti \& S. Chatterjee 03-001715 (LWG 15371). Uttarakhand, Pithoragarh district, Milam, en route to Milam glacier, alt. $3434 \mathrm{~m}$, on R. chrysoleuca colonizing rocks, 21 Oct 2007, Santosh Joshi 07-010418 (LWG 11551); Martoli, en route to Milam 
glacier, alt. $3390 \mathrm{~m}$, on $R$. chrysoleuca colonizing rocks, 23 Oct 2007, Santosh Joshi 07-010540 (LWG); Mapan to Rilkot, en route to Milam glacier, alt. 3160-3200 m, on P. muralis colonizing exposed rocks, 19 Oct 2007, Santosh Joshi 07-010357 (LWG).

L. usneae (Anzi) D. Hawksw., Persoonia 9 (2): 185 (1977)

Host - Flavoparmelia caperata

Known distribution - Jammu \& Kashmir.

Notes - The species was reported from Jammu \& Kashmir by Zhurbenko (2013).

L. xanthoriae M.S. Christ., Friesia 5 (3-5): 212 (1956)

Hosts - Melanelixia subargentifera, Xanthoria elegans

Known distribution - Jammu \& Kashmir, Uttarakhand.

Material examined - India, Uttarakhand, Chamoli district, 1/2 km before Malari, on way to Niti, alt. 3086 m, on X. elegans colonizing rocks, 19 Aug 2007, Upreti \& Nayaka 07-011223 (LWG).

Notes - The species was previously reported from Jammu \& Kashmir by Zhurbenko (2013) and in the present study; it is extending its distributional range up to Uttarakhand where it is colonizing X. elegans.

LICHENODIPLIS Dyko \& D. Hawksw., The Lichenologist 11 (1): 51 (1979)

L. lecanorae (Vouaux) Dyko \& D. Hawksw., The Lichenologist 11 (1): 52 (1979)

Hosts - Acarospora sp., Caloplaca cerina, Lecanora allophana, L. somervellii, L. tropica, Porpidia crustulata, P. hydrophila, Pertusaria leioplacella, Rhizocarpon geographicum, Xanthoria candelaria, X. elegans

Known distribution - Himachal Pradesh, Jammu \& Kashmir, Karnataka, Madhya Pradesh, Uttarakhand.

Materials examined - India, Himachal Pradesh, Kangra district, Bir tea garden area, Billing hills, alt. 2500 m, on L. allophana colonizing Quercus tree bark, 02 Jun 1994, D.K. Upreti 213617 (LWG 15238); Kullu district, Great Himalayan National Park, around Patal, alt. $2800 \mathrm{~m}$, on $P$. hydrophila colonizing exposed rocks, 07 Sept 1999, D.K. Upreti 99-53657 (LWG 21203); on L. somervellii colonizing exposed rocks, 07 Sept 1999, D.K. Upreti 99-53665/B (LWG 15974); Lahul Spiti district, Spiti, Spiti valley, Rongrick, alt. 3600 m, on X. elegans colonizing rocks, 04 Aug 2002, Upreti \& Divakar 02-000109 (LWG); Lossar, alt. 3900 m, on Acarospora sp. colonizing rocks, 04 Aug 2002, Upreti \& Divakar 02-00094 (LWG). Jammu \& Kashmir, Gulmarg district, at Gulmarg on southeast side, alt. 2560-2800 m, on P. crustulata colonizing boulders, 02 Jul 1977, K. Dange 77.464 (LWG-LWU). Karnataka, Hassan district, near Sakleshpuram-Sambhalli, alt. 800 m, on P. leioplacella colonizing mango tree bark, 01 May 1979, D.D. Awasthi \& P. Srivastava 79-316 (LWG-LWU). Madhya Pradesh, Katni district, Khitauli, near Kalwera village, on Lecanora sp. growing along with $P$. leucostoma colonizing bark of Shorea robusta, 20 Feb 2009, G.K. Mishra \& S. Mohabe 09-009930/C (LWG 10567); Mandla district, Kanha National Park, Kisli area, on $L$. tropica colonizing twigs, 12 Oct 2004, D.K. Upreti \& Yogesh Joshi 04-004503 (LWG 1534). Uttarakhand, Chamoli, on $R$. geographicum colonizing exposed rocks, 21 Aug 2007, D.K. Upreti \& S. Nayaka 07-010138 (LWG); Almora district, G.B. Pant Institute of Himalayan Environment and Development, on apothecia of Lecanora sp. colonizing bark of Ligustrum nepalense, 16-18 Mar 2014, G. Bhakuni s.n. (KU).

Notes - The species was previously reported from Jammu \& Kashmir and Uttarakhand by Zhurbenko (2013) and Joshi et al. (2015a) respectively, and in the present study; it is extending its distributional range up to Himachal Pradesh, Karnataka and Madhya Pradesh. It is one of the most common species of lichenicolous fungi in India. Rhizocarpon geographicum is the new host for this species. 
L. lichenicola Dyko \& D. Hawksw., The Lichenologist 11 (1): 56 (1979)

Host - Rinodina sp.

Known distribution - Uttarakhand.

Material examined - Uttarakhand, Champawat district, Shivalaya temple, on apothecia of Rinodina sp. colonizing rocks, 17 Jul 2014, K. Chandra s.n. (KU).

LICHENOPELTELLA Höhn., Sitzungsberichte der Kaiserlichen Akademie der Wissenschaften Math.-naturw. Klasse Abt. I 128: 553 (1919)

L. swaminathaniana Harih., Mibey \& D. Hawksw., The Lichenologist 28 (3): 294 (1996)

Host - Porina sp.

Known distribution - Tamil Nadu.

Notes - The species was reported from Tamil Nadu by Hariharan et al. (1996).

LICHENOSTIGMA Hafellner, Herzogia 6 (1-2): 301 (1983)

L. alpinum (R. Sant., Alstrup \& D. Hawksw.) Ertz \& Diederich, Fungal Diversity 66: 126 (2014)

Host - Pertusaria albescens

Known distribution - Jammu \& Kashmir.

Notes - The species was reported from Jammu \& Kashmir by Zhurbenko (2013).

L. cosmopolites Hafellner \& Calat., Mycotaxon 72: 108 (1999)

Hosts - Xanthoparmelia australasica, X. congensis, X. conspersa, X. coreana, X. mexicana, $X$. pseudocongensis, X. stenophylla, X. terricola

Known distribution - Himachal Pradesh, Jammu \& Kashmir, Rajasthan, Sikkim, Uttarakhand.

Materials examined - India, Himachal Pradesh, Kinnaur district, Recong Peo, in and around Kalpa, alt. 2950 m, on X. stenophylla colonizing rocks, 03 Nov 2003, Upreti, Srivastava \& Prakash 03-002651/B (LWG 25894); alt. 4000 m, on X. australasica colonizing rocks, 03 Nov 2003, Upreti, Srivastava \& Prakash 03-002657 (LWG); Racksham to Chitkul, alt. 3500 m, on X. stenophylla colonizing soil over rocks in moist places, 05 Nov 2003, Upreti, Srivastava \& Prakash 03-002773 (LWG 25891); Lahaul Spiti district, Zinzibar, alt. 4200 m, on X. stenophylla colonizing exposed rocks, 04 Aug 2003, D.K. Upreti \& S. Chatterjee 03-001757 (LWG 25939); Shimla district, Rohru, Chirgaon, Sandasu, alt. 1700 m, on X. australasica colonizing rocks, 19 May 2002, S. Nayaka \& R. Srivastava 02-81669 (LWG). Jammu \& Kashmir, Doda district, Kistwar, Sarthal forest area, alt. $2100 \mathrm{~m}$, on X. mexicana colonizing exposed rocks, 03 Nov 2005, Rohini Rana s.n. (LWG 25973); Kukernag, alt. $2438 \mathrm{~m}$, on X. conspersa colonizing rocks, 21 Mar 1970, P.N. Mujoo 70.52 A (LWG-LWU). Rajasthan, Sirohi district, Mount Abu, alt. $1680 \mathrm{~m}$, on X. pseudocongensis colonizing rocks, 15 Jan 1973, A. Singh 101470 (LWG). Sikkim, North Sikkim district, Giagaon, above Jhanngu, alt. $4600 \mathrm{~m}$, on X. stenophylla colonizing soil, 13 Aug 2004, D.K. Upreti, S. Chatterjee \& P.K. Divakar 04-004017 (LWG 26012); Kalep, before Thangu, alt. 3900 m, on X. mexicana colonizing soil, 12 Aug 2004, D.K. Upreti, S. Chatterjee \& P.K. Divakar 04-003837 (LWG 25928); Thangu area, alt. $4000 \mathrm{~m}$, on X. mexicana colonizing rocks, D.K. Upreti, S. Chatterjee \& P.K. Divakar 04-003896 (LWG 25974); Chungthag, Thangu, Chopta valley, alt. 3682

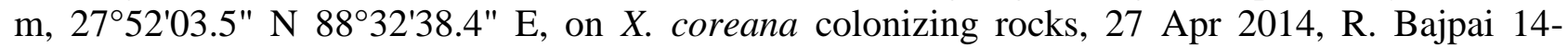
022973 (LWG 29102). Thangu, alt. $3682 \mathrm{~m}$, on X. mexicana colonizing rocks, 27 Apr 2014, R. Bajpai 14-024309 (LWG 30329). Uttarakhand, Almora district, Almora, alt. $1524 \mathrm{~m}$, on $X$. conspersa colonizing stones of walls, 14 Jun 1956, D.D. Awasthi 3522 (LWG-AWAS); Chamoli district, alt. $1350 \mathrm{~m}$, on X. congensis colonizing rocks, 20 Oct 1967, A. Singh \& party 91544 (LWG); way to Niti, $10 \mathrm{~km}$ before Niti, alt. $3118 \mathrm{~m}$, on X. conspersa colonizing rocks, 20 Aug 2007, D.K. Upreti \& S. Nayaka 07-010297 (LWG 25967); Jumma, alt. $2800 \mathrm{~m}$, on $X$. pseudocongensis and X. terricola colonizing soil over rocks, 24 Sept 2006, S. Rawat 06-006815 
(LWG 25968); alt. $3200 \mathrm{~m}$, on X. stenophylla colonizing rocks, 01 Jul 2007, S. Rawat 07-008666 (LWG 26024); Badrinath, between Vasudhara and Bhagirathi Glacier, alt. 3900-4500 m, on thallus of X. stenophylla colonizing exposed soil, 09 Sept 1991, D.K. Upreti L13209 (LWG 25924); near Vasudhara glacier, alt. $3900 \mathrm{~m}$, on X. stenophylla colonizing soil, 08 Sept 1991, D.K. Upreti 202379 (LWG 25922); way to Niti, $10 \mathrm{~km}$ before Niti, alt. $3118 \mathrm{~m}$, on thallus of X. stenophylla colonizing exposed rocks, 20 Aug 2007, D.K. Upreti \& S. Nayaka 07-010289 (LWG 25963); Badrinath, south of temple, near Brahmini village, alt. 3125-3200 m, on X. conspersa colonizing boulders, 27 Sept 1976, K. Dange 76-762 (LWG 25912); east of temple, on way to Dhantoli village, alt. ca. $3250 \mathrm{~m}$, on X. mexicana colonizing boulders along mosses, 29 Sept 1976, K. Dange 76.850 (LWG-LWU); Mana village, towards Vasudhara glacier, on X. stenophylla colonizing rocks, Aug 2003, S.M. Singh 03-001829 (LWG 25940); Vasudhara Glacier, on X. stenophylla colonizing rocks, Aug 2003, S.M. Singh 03-001842 (LWG 26010); Mana village, alt. 3350 m, on $X$. stenophylla colonizing rocks, 21 Jun 2005, V. Shukla \& Y. Joshi 05-005307 [DUP] (LWG 25884); behind Garhwal Jal Sansthan Gust House, on X. stenophylla colonizing rocks, 21 Jun 2005, V. Shukla \& Y. Joshi , 05-005352, 05-005354 (LWG 25958); Pithoragarh district, between Milam and Martoli, alt. $3000 \mathrm{~m}$, on Xanthoparmelia sp. growing along with Xanthoria elegans colonizing rocks, 01 Jul 1973, Ajay Singh 102845 (LWG); Milam glacier, alt. 3450 m, on X. stenophylla colonizing rocks, 29 Jun 1973, A. Singh 102823, 102825 (LWG 25982); Uttarkashi district, Gomukh area, right bank, $5^{\text {th }}$ moraine, alt. ca. $3840 \mathrm{~m}$, on X. australasica colonizing rocks, $04 \mathrm{Jul}$ 1976, D.D. Awasthi \& S.R. Singh 8500 (LWG-AWAS 15715); on way to Gangotri, $3.5 \mathrm{~km}$ from Bhairown Ghati, alt. ca. $3050 \mathrm{~m}$, on X. mexicana colonizing rocks, 29 Jun 1976, D.D. Awasthi \& S.R. Singh 8162 (LWG-AWAS 15890); Gangotri, alt. $3260 \mathrm{~m}$, on X. conspersa colonizing rocks, 26 Sept 1977, Ajay Singh \& Ram Phir 75375 (LWG 25917); on X. stenophylla colonizing rocks, 26 Sept 1977, Ajay Singh 75377 (LWG 26011); alt. 3120 m, on X. stenophylla colonizing rocks, 26 Sept 1977, Ajay Singh s.n. (LWG 25886); near Gangotri, towards Gomukh, alt. 3200 m, on X. australasica colonizing rocks, 30 Jun 1976, D.D. Awasthi \& S.R. Singh 8222 (LWG-AWAS 15214); en route from Gangotri to Chirwasa, alt. $3204 \mathrm{~m}, 30^{\circ} 59^{\prime} 47.7^{\prime \prime} \mathrm{N} 78^{\circ} 58^{\prime} 17.3^{\prime \prime} \mathrm{E}$, on $X$. terricola colonizing soil on rocks, 27 Oct 2010, Himanshu Rai \& Pramod Nag 10-0014501 (LWG 26041); $2 \mathrm{~km}$ before Chirwasa, on $X$. stenophylla colonizing rocks, 05 Sept 2002, S. Chatterjee \& P.K. Divakar 02-000180, 02-000181 (LWG 25980; 25983); Chirwasa, alt. $3500 \mathrm{~m}$, on $X$. stenophylla colonizing rocks, 07 Sept 2002, S. Chatterjee \& P.K. Divakar 02-000242 (LWG 25981).

Notes - It is the most common species of lichenicolous fungi in India and was previously reported from Jammu and Kashmir (Zhurbenko 2013). In the present study, it is extending its distribution in different states of India.

L. cf. elongatum Nav.-Ros. \& Hafellner, Mycotaxon 57: 213 (1996)

Hosts - Lecanora sp., Lobothallia praeradiosa

Known distribution - Jammu \& Kashmir.

Notes - The species was reported from Jammu \& Kashmir by Zhurbenko (2013).

*L. maureri Hafellner, Kew Bulletin Additional Series 9: 301 (1983)

Host - Xanthoparmelia stenophylla

Known distribution - Jammu \& Kashmir.

Material examined - India, Jammu \& Kashmir, between Pahalgam and Lidderwat, on $X$. stenophylla, 28 May 1954, K.N. Kaul s.n. (LWG 25934).

*L. triseptatum Halici \& D. Hawksw., The Lichenologist 39 (5): 439 (2007)

Hosts - Aspicilia almoneresis, A. verruculosa, Rhizocarpon disporum

Known distribution - Himachal Pradesh, Uttarakhand.

Materials examined - India, Himachal Pradesh, Kangra district, Puti Runi, on A. verruculosa colonizing boulders, 14 Aug 1952, O.A. Höeg 1737 (LWG-AWAS 000549); Lahul 
Spiti district, Lahul valley, Chhatru, alt. $3200 \mathrm{~m}$, on A. almorensis colonizing exposed rocks, 03 Aug 2002, D.K. Upreti \& P.K. Divakar 02-000066 (LWG 005192); Kinnaur district, in and around Yangthang, alt. $3800 \mathrm{~m}$, on $R$. disporum colonizing rocks, s.d., Upreti, Srivastava \& Prakash 03002636 (LWG 23266). Uttarakhand, Uttarkashi district, Gomukh area, right bank, $2^{\text {nd }}$ moraine, on A. verruculosa colonizing rocks, 02 Jul 1976, D.D. Awasthi \& S.R. Singh 8401 (LWG-AWAS 0004612).

Notes - Rhizocarpon disporum is the new host for this species.

LICHENOTHELIA D. Hawksw., The Lichenologist 13(2): 142 (1981)

*L. convexa Henssen, Bibliotheca Lichenologica 25: 259 (1987)

Host - Aspicilia dwaliensis

Known distribution - Uttarakhand.

Material examined - India, Uttarakhand, Tehri Garhwal, Jamuna Valley, near Kharsali, alt. $2135 \mathrm{~m}$, on A. dwaliensis colonizing rocks, 20 Jun 1951, D.D. Awasthi 970 (LWG-AWAS 000478).

MARCHANDIOMYCES Diederich \& D. Hawksw., Mycotaxon 37: 311 (1990)

M. corallinus (Roberge) Diederich \& D. Hawksw., Mycotaxon 37: 312 (1990)

Hosts - Physcia aipolia, Xanthoria candelaria

Known distribution - Jammu \& Kashmir.

Notes - The species was reported from Jammu \& Kashmir by Zhurbenko (2013).

MELASPILEA Nyl., Actes de la Société Linnéenne de Bordeaux 21: 416 (1857)

M. amarkantakensis S. Joseph \& G.P. Sinha, Taiwania 60 (1): 18 (2015)

Host - Pertusaria amarkantakana

Known distribution - Madhya Pradesh.

Notes - The species was reported from Madhya Pradesh by Joseph \& Sinha (2015).

\section{M. insitiva Stirt. (1879)}

Host - Pertusaria sp.

Known distribution - West Bengal, Uttarakhand.

Material examined - India, Uttarakhand, Almora district, Kausani, Kantli village, near Rudra Dhari temple, on thallus of Pertusaria sp., 01 Apr 2015, Pooja Joshi \& Pooja Chilwal s.n. (KU).

Notes - The species was previously reported from West Bengal by Joseph \& Sinha (2015) and in the present study; it is extending its distributional range up to Uttarakhand.

MONEROLECHIA Trevis., Linnaea 28: 296 (1857)

*M. badia (Fr.) Kalb, Bibliotheca Lichenologica 88: 312 (2004)

Hosts - Acarospora smaragdula, Aspicilia almorensis, A. caesiocinerea

Known distribution - Himachal Pradesh, Sikkim, Uttarakhand.

Materials examined - India, Himachal Pradesh, Kullu district, Great Himalayan National Park, around Shilt, alt. $3000 \mathrm{~m}$, on thallus of A. smaragdula colonizing exposed rocks, 05 Sept 1999, D.K. Upreti 99-53618 (LWG); ibid., alt. 2600-3000 m, on thallus of A. smaragdula colonizing exposed rocks, 06 Sept 1999, D.K. Upreti 99-53620 (LWG); Ordi, alt. 2100-2170 m, on thallus of A. almorensis colonizing rocks, 06 Nov 2002, S. Nayaka \& R. Srivastava 02-001071 (LWG 005227); around Shilt, alt. 2600-3000 m, on thallus of A. almorensis colonizing exposed rocks, 05 Sept 1999, D.K. Upreti 99-52686 (LWG 005189); Shimla district, Rohru, Chirgaon, 
Tikkri, alt. $1700 \mathrm{~m}$, on thallus of A. almorensis colonizing rocks, 21 May 2002, S. Nayaka \& R. Srivastava 02-213889 (LWG 0059183). Sikkim, North Sikkim, above Lachung, Shingringtang, alt. $2900 \mathrm{~m}$, on thallus of A. almorensis colonizing rocks, 16 Aug 2004, D.K. Upreti, S. Chatterjee \& P.K. Divakar 04-004296 (LWG 005231). Uttarakhand, Chamoli district, way of Nanda Devi Biosphere Reserve, Lata, alt. 2900 m, on thallus of A. almorensis colonizing rocks, 05 Jun 2008, S. Rawat 08-011163 (LWG 008018); Pithoragarh district, Lilam to Bogudiyar, en route to Milam glacier, alt. 1800-2450 m, on thallus of A. caesiocinerea colonizing rocks, 17 Oct 2007, S. Joshi 07-010527 (LWG 11671); Bogudiyar-Naher Devi, Milam glacier, alt. 2400-2705 m, on thallus of A. almorensis colonizing rocks, 18 Oct 2007, S. Joshi, 07-010575/A \& 07-010768 (LWG 11687 \& 11718 respectively).

Notes - Acarospora smaragdula is a new host for this lichenicolous lichen.

MONODICTYS S. Hughes, Canadian Journal of Botany 36 (6): 785 (1958)

M. epilepraria Kukwa \& Diederich, The Lichenologist 37 (3): 217 (2005)

Host - Lepraria sp.

Known distribution - Jammu \& Kashmir.

Notes - The species was reported from Jammu \& Kashmir by Zhurbenko (2013).

MUELLERELLA Hepp ex Müll. Arg., Mémoires de la Société de Physique et d'Histoire Naturelle de Genève 16 (2): 419 (1862)

M. erratica (A. Massal.) Hafellner \& V. John, Herzogia 19: 165 (2006)

Hosts - Acarospora badiofusca, A. bullata, A. fuscata, A. veronensis, Aspicilia caesiocinerea, A. maculata, Pleopsidium flavum, Protoparmeliopsis muralis, Lecidea lapicida, Lobothallia alphoplaca, L. praeradiosa, Pertusaria sp., Rhizoplaca melanophthalma, Xanthoria elegans

Known distribution - Himachal Pradesh, Jammu \& Kashmir, Uttarakhand.

Materials examined - India, Himachal Pradesh, Brahmaur Hadsar, Mani Mahesh, Doonch, alt. 1600-2700 m, on A. almorensis colonizing rocks, 16 May 2001, D.K. Upreti \& S. Nayaka 0175490/A (LWG 005275); Kinnaur district, in and around Yangthang, alt. $3800 \mathrm{~m}$, on A. fuscata colonizing rocks, 02 Nov 2003, Upreti, Srivastava \& Prakash 03-0026361 (LWG); in and around Puh, alt. $3000 \mathrm{~m}$, on L. alphoplaca colonizing rocks, 01 Nov 2003, Upreti, Srivastava \& Prakash 03-002605/B (LWG 005318); in and around Nako lake area, alt. $3950 \mathrm{~m}$, on L. alphoplaca colonizing rocks, 02 Nov 2003, Upreti, Srivastava \& Prakash 03-002619/C (LWG 005323); Lahul Spiti district, Rohtang Pass area, alt. $3600 \mathrm{~m}$, on P. muralis colonizing exposed rocks, 13 Sept 2001, D.K. Upreti 01-26514/A (LWG 15608); Zinzibar, alt. 4200 m, on L. praeradiosa colonizing exposed rocks, 04 Aug 2003, D.K. Upreti \& S. Chatterjee 03-001763 (LWG 005315); Lahul valley, Chhatru, alt. 3200 m, on P. muralis colonizing rocks, 03 Aug 2002, Upreti \& Divakar 02-000058/H (LWG 15557); on L. alphoplaca colonizing rocks, 03 Aug 2012, Upreti \& Divakar 02-000057/C (LWG 005144); near Chhatru, alt. 3900 m, on P. muralis colonizing rocks, 06 Aug 2002, Upreti \& Divakar 02-000166 (LWG 15559); Spiti valley, Lithang, alt. 3600 m, on A. fuscata colonizing exposed rocks, 05 Aug 2002, D.K. Upreti \& P.K. Divakar 02-000118 (LWG); Spiti-Chaudra valley, Puti Runi, alt. 3500 m, on A. bullata colonizing boulders, 14 Aug 1952, O.A. Höeg 1731 (LWG-AWAS 2053). Jammu \& Kashmir, Ladakh, Hemis National Park, Rumbak valley, Gandala 2, on P. muralis colonizing rocks, 28 Jul 1999, H.R. Negi L25F (CES) (LWG 15925); on $X$. elegans colonizing rocks, 28 Jul 1999, H.R. Negi L22 (CES) (LWG); Suru, Panikar, Suru valley, on A. maculata colonizing rocks, 12 Sept 2012, Jatinder Kumar 019413 (LWG 13215); Zanskar, Ichar, on A. maculata colonizing rocks, 08 Jul 2012, Jatinder Kumar 12-019273 (LWG 13355); Leh District, Changla, on A. caesiocinerea colonizing rocks, 05 Jul 2010, Jatinder Kumar 10-014333 (LWG 10408); on R. melanophthalma colonizing rocks, 05 Jul 2010, Jatinder Kumar 10-014337 (LWG); Pahalgam, on way from Chandanwari to Sheshnag up to Pissu top, alt. 2700-3360 m, on 
Pertusaria growing along with P. muralis colonizing rocks, 30 Jun 1977, K. Dange 77.390 (LWGLWU). Uttarakhand, Bageshwar district, near Pindari glacier, Mirtoli, alt. $3600 \mathrm{~m}$, on A. veronensis colonizing sloping boulders, 11 Jun 1970, D.D. Awasthi 7709 (LWG-AWAS 000133); Chamoli district, near Bardinath, way from Mana to Vasudhara, alt. $3350 \mathrm{~m}$, on P. flavum colonizing exposed rocks, 21 Aug 2007, D.K. Upreti \& S. Nayaka 07-010114 (LWG 005731); Badrinath, east of temple, on way to Dhantoli village, alt. $3250 \mathrm{~m}$, on P. flavum colonizing boulders, 29 Sept 1976, K. Dange 76.882 (LWG-LWU 000119); Uttarkashi district, GWLS, Osla, near hanging bridge, alt. $3037 \mathrm{~m}, 31^{\circ} 07^{\prime} 44.26^{\prime \prime} \mathrm{N} 78^{\circ} 22^{\prime} 40.86^{\prime \prime} \mathrm{E}$, on A. badiofusca colonizing rocks, 11 Jun 2012, D.K. Upreti \& R. Bajpai 12-016193 (LWG).

Notes - The species was previously reported from Jammu \& Kashmir by Zhurbenko (2013) and in the presnt study it is extending its distributional range up to Himachal Pradesh and Uttarakhand.

*M. lichenicola (Sommerf.) D. Hawksw., Botaniska Notiser 132: 289 (1979)

Hosts - Lecanora sp., Psora decipiens, Psora sp., Xanthoria elegans

Known distribution - Himachal Pradesh, Jammu \& Kashmir, Uttarakhand.

Materials examined - India, Himachal Pradesh, Parbati river valley, on way to Pulga from Manikaran, just before bridge on Parbati, alt. 1950 m, on Psora colonizing rocks, 18 Jun 1975, D.D. Awasthi \& K. Dange 75095 (LWG 13458); Lahul Spiti district, Spiti valley, Zinzibar, alt. 4200 m, on Lecanora colonizing exposed rocks, 04 Aug 2003, D.K. Upreti \& S. Chatterjee 03001761/A (LWG 23391). Jammu \& Kashmir, Leh district, Fyang, alt. $3660 \mathrm{~m}$, on X. elegans colonizing rocks, 19 Jun 2010, Jatinder Kumar 10-14311 (LWG); Leh, alt. ca. $3520 \mathrm{~m}$, on X. elegans colonizing rocks, 12 Jun 2010, Jatinder Kumar 10-014301 (LWG). Uttarakhand, Pithoragarh district, Rilkot to Burfu, en route to Milam glacier, alt. 3200-3250 m, on P. decipiens colonizing soil, s.d., Santosh Joshi 07-010353 (LWG).

Notes - Psora decipiens is the new host for this fungus.

M. pygmaea (Körb.) D. Hawksw., Botaniska Notiser 132: 289 (1979)

Hosts - Acarospora sp., Xanthoria elegans

Known distribution - Jammu \& Kashmir.

Notes - The species was reported from Jammu \& Kashmir by Zhurbenko (2013).

*M. ventosicola (Mudd) D. Hawksw., The Lichenologist 35 (3): 212 (2003)

Hosts - Rhizocarpon disporum, $R$. geographicum

Known distribution - Jammu \& Kashmir, Uttarakhand.

Materials examined - India, Jammu \& Kashmir, Leh, Rangdum, on $R$. disporum colonizing rocks, s.d., Jatinder Kumar 12-021249 (LWG). Uttarakhand, Chamoli district, Badrinath, east of temple, on way to Dhantoli village, alt. ca. $3250 \mathrm{~m}$, on $R$. geographicum colonizing boulders, 29 Sept 1976, K. Dange 76.859 (LWG-LWU 05846).

NECTRIOPSIS Maire, Annales Mycologici 9: 323 (1911)

N. lecanodes (Ces.) Diederich \& Schroers, Lejeunia 162: 56 (1999)

Hosts - Lobaria pseudopulmonaria, Peltigera elisabethae, P. scabrosa

Known distribution - Jammu \& Kashmir, Sikkim.

Material examined - India, Sikkim, North Sikkim, near Yumthang, alt. 3800 m, on thallus of L. pseudopulmonaria colonizing exposed tree trunk, 15 Aug 2004, D.K. Upreti, S. Chatterjee \& P.K. Divakar 04-004160/A (LWG 13027).

Notes - The species was previously reported from Jammu \& Kashmir by Zhurbenko (2013), where it was colonizing Peltigera species. In the present study, the species is extending its distributional as well as host range. 
NESOLECHIA A. Massal., Miscellanea lichenologica: 43 (1856)

N. fusca (Triebel \& Rambold) Pérez-Ortega, The Bryologist 113 (3): 487 (2010)

Hosts - Canoparmelia sp., Xanthoparmelia conspersa, X. pseudocongensis, X. stenophylla

Known distribution - Uttarakhand.

Materials examined - India, Uttarakhand, Chamoli district, Malari area, alt. $2400 \mathrm{~m}$, on Canoparmelia sp. colonizing rocks, 24 Sept 2006, S. Rawat 06-006768 (LWG 23418); Jumma area, alt. $2800 \mathrm{~m}$, on X. pseudocongensis colonizing rocks, 24 Sept 2006, S. Rawat 06-006815 (LWG); Uttarkashi district, Chirwasa, alt. $3500 \mathrm{~m}$, on X. conspersa colonizing rocks, 07 Sept 2002, S. Chatterjee \& P.K. Divakar 02-000242/B (LWG 25920); on way to Gangotri, $6 \mathrm{~km}$ from Bhairown ghati, alt. ca. 3100 m, on X. stenophylla colonizing boulders, 29 Jun 1976, D.D. Awasthi \& S.R. Singh 8198 (LWG-AWAS 15712).

ODONTOTREMA Nyl., Mémoires de la Société Impériale des Sciences Naturelles de Cherbourg 5: 143

*O. pertusariae Etayo, Diederich \& Coppins, The Lichenologist 34 (6): 494 (2002)

Host - Pertusaria leucostoma

Known distribution - Madhya Pradesh.

Material examined - India, Madhya Pradesh, Rewa district, Baikunthpur, Khamariya, on thallus of Pertusaria leucostoma colonizing Acacia tree, 18 Feb 2009, G.K. Mishra \& S. Mohabe 09-009134/B (LWG 10527).

OPEGRAPHA Ach., Kongliga Vetenskaps Academiens Nya Handlingar 30: 97 (1809)

*O. brigantina Hafellner, Herzogia 7 (1-2): 173 (1985)

Host - Brigantiaea leucoxantha

Known distribution - Uttarakhand.

Material examined - India, Uttarakhand, Jim Corbett Tiger Reserve, on thallus of $B$. leucoxantha colonizing exposed Syzygium cuminii bark, 03 Dec 1999, D.K. Upreti \& Jyoti Tandon 217455 (LWG 001114).

O. foreaui (C. Moreau \& M. Moreau) Hafellner \& R. Sant., Fritschiana 36: 14 (2002)

Hosts - Heterodermia diademata, $H$. incana, $H$. japonica, $H$. leucomelos, $H$. pseudospeciosa, $H$. speciosa, $H$. togashii

Known distribution - Kerala.

Materials examined - India, Kerala, Idukki district, Kanthalloor, Mannavan Shola, alt. ca. 2000 m, on Heterodermia sp. colonizing bark, 13 Jan 1999, Stephen 21961 (KFRI); Periyar Tiger Reserve, Uppupara, alt. ca. 1150 m, on Heterodermia sp. colonizing rocks, 12 Dec 1998, Stephen 21839 (KFRI); Kottayam district, Parathodu, alt. 900 m, on H. diademata, s.d., Stephen 23293 (KFRI); Palakkad district, Silent Valley National Park, Anginda, alt. 2200 m, on H. togashii, s.d., Stephen s.n. (KFRI); ibid., on H. incana, s.d., Stephen s.n. (KFRI); ibid., on H. japonica, s.d., Stephen s.n. (KFRI); Sispara, alt. 2050 m, on H. togashii, s.d., Stephen, s.n. (KFRI); s.loc., on $H$. pseudospeciosa, s.d., Stephen s.n. (KFRI); Palghat district, Siruvani, Dam site, alt. ca. 750 m, on $H$. pseudospeciosa colonizing rocks, 10 Nov 1998, Stephen 2116 (KFRI); Pathanamthitta district, Kakki, Dam site, Kakki, alt. ca. 900 m, on H. pseudospeciosa colonizing rocks, 09 Dec 1998, Stephen 21807 (KFRI).

Notes - The species was reported from Manaloor region of Kerala by Moreau (1951a) and is rediscovered after a gap of fifty-five years from Kerala.

O. phaeophysciae R. Sant., Diederich, Ertz \& Christnach, Bibliotheca Lichenologica 91: 132 (2005) 
Host - Phaeophyscia hispidula

Known distribution - Uttarakhand.

Materials examined - India, Uttarakhand, Pithoragarh district, Thal Ke Dhar, alt. 2082 m, 29 30'57.7" N, 80 14'20.5" E, over, over P. hispidula colonizing bark of Quercus, 05 Aug 2014, Shashi Upadhyay \& Sandhya Shukla (PUN 7154); ibid., on way to Asurchula temple (Chana village), alt. 2000 m, 29 37'47.42" N, 80 10'38.66" E, over P. hispidula colonizing bark of Quercus, 06 Aug 2014, Shashi Upadhyay \& Sandhya Shukla (PUN 7153); Chandak Pashupati Nath forest, Mosta Manu temple, alt. 1800 m, 29 36'53.60" N, 80 11'48.31" E, over P. hispidula colonizing bark of Quercus, 07 Aug 2014, Shashi Upadhyay \& Sandhya Shukla (PUN 7152).

O. trassii S.Y. Kondr. \& Coppins, Folia Cryptogamica Estonica 32: 10 (1998)

Host - Heterodermia. leucomelos

Known distribution -Tamil Nadu.

Notes - The species reported from Chennai district of Tamil Nadu by Coppins \& Kondratyuk (1998) was missing in the preliminary checklists of Joshi et al. (2015a) and Zhurbenko (2013). Both of them cited the wrong reference for $O$. foreaui, which was reported from Kerala by Moreau (1951a). To one of the authors (YJ), O. trassii seems similar to O. foreaui, but it will only be confirmed after visualizing the type specimens of both the species.

PHOMA Sacc., Michelia 2 (6): 4 (1880)

\section{Phoma sp.}

Host - Xanthoria elegans

Known distribution - Jammu \& Kashmir, Uttarakhand.

Notes - The species was previously reported from Jammu \& Kashmir by Zhurbenko (2013). The same sort of characters of Phoma were found by the authors in a sample of $X$. elegans collected from Uttarakhand, but as the specimen was poor we aren't describing it as something new.

PHYLLOSTICTA Pers., Traité sur les Champignons Comestibles: 147 (1818)

P. galligena Moreau, Bulletin de la Société botanique de France: 102 (1951)

Host - Parmotrema perforatum

Known distribution - Tamil Nadu.

Notes - The species was reported from Tamil Nadu by Moreau (1951b).

POLYCOCCUM Saut. ex Körb., Parerga lichenologica. Ergänzungen zum Systema lichenum Germaniae: 470 (1865)

P. clauzadei Nav.-Ros. \& Cl. Roux, Mycotaxon 69: 329 (1998)

Host - Xanthoria elegans

Known distribution - Jammu \& Kashmir, Uttarakhand.

Materials examined - India, Uttarakhand, Chamoli district, from Mana to Vasudhara, alt. $3300 \mathrm{~m}$, on X. elegans colonizing rocks, 21 Aug 2007, Upreti \& S. Nayaka 07-011227 (LWG); Pithoragarh district, Martoli, en route to Milam glacier, alt. 3390 m, on X. elegans colonizing rocks, 23 Oct 2007, S. Joshi 07-010540 (LWG).

Notes - The species was previously reported from Jammu \& Kashmir by Zhurbenko (2013) and in the present study; it is extending its distributional range up to Uttarakhand.

*P. microsticticum (Leight.) Arnold, Berichte der Bayerischen Botanischen Gesellschaft 1 (suppl.): 132 (1891)

Host - Acarospora fuscata

Known distribution - Himachal Pradesh. 
Material examined - India, Himachal Pradesh, Lahul Spiti district, Spiti valley, Lithang, alt. 3400 m, on A. fuscata colonizing exposed rocks, 05 Aug 2002, D.K. Upreti \& P.K. Divakar 02000116/A (LWG)

*P. peltigerae (Fuckel) Vězda, Ceská Mykologie 23 (2): 109 (1969)

Host - Peltigera canina

Known distribution - Uttarakhand.

Material examined - India, Uttarakhand, Bageshwar district, en route to Pindari glacier from Dhakuri to Khati, alt. 2083-2210 m, on P. canina colonizing soil, 11 May 2007, Yogesh Joshi \& Santosh Joshi 07-008816 (LWG).

P. pulvinatum (Eitner) R. Sant., Lichens and Lichenicolous Fungi of Sweden and Norway: 175 (1993)

Host - Physcia dubia

Known distribution - Jammu \& Kashmir.

Notes - The species was reported from Jammu \& Kashmir by Zhurbenko (2013).

POLYSPORINA Vězda, Folia Geobotanica et Phytotaxonomica 13 (4): 399 (1978)

*P. subfuscescens (Nyl.) K. Knudsen \& Kocourk., Mycotaxon 105: 151 (2008)

Hosts - Candelariella aurella, Carbonea sp.

Known distribution - Jammu \& Kashmir.

Materials examined - India, Jammu \& Kashmir, Ladakh, Chungthang valley, Souiriri, 20 $\mathrm{km}$ before Souiriri, on Carbonea sp. growing along with Xanthoria elegans on rocks, 02 Aug 2012, Jatinder Kumar 12-021242 (LWG); Leh district, Ganglar area, alt. 4000 m, on Carbonea sp. growing along with Acarospora sp. on exposed rocks, 08 Aug 2003, D.K. Upreti \& S. Chatterjee 03-001823 (LWG 00594); Srinagar, on C. aurella colonizing soil, 1960, G.C. Rath s.n. (LWG 007477).

PRONECTRIA Clem., The genera of Fungi: 78, 282 (1931)

P. subimperspicua (Speg.) Lowen, Mycotaxon 39: 462 (1990)

Host - Punctelia borreri

Known distribution - Jammu \& Kashmir.

Notes - The species was reported from Jammu \& Kashmir by Zhurbenko (2013).

PYRENIDIUM Nyl., Flora (Regensburg) 48: 210 (1865)

P. actinellum Nyl., Flora (Regensburg) 48: 210 (1865)

Hosts - Peltigera canina, $P$. elisabethae, $P$. praetextata, Punctelia rudecta

Known distribution - Jammu \& Kashmir, Uttarakhand.

Materials examined - India, Uttarakhand, Almora district, Syahi Devi Forest, on thallus of P. rudecta colonizing Quercus tree, 27 Jul 2014, S. Upadhyay \& N. Rana, s.n. (KU); Bageshwar district, alt. 2210-2734 m, on P. canina colonizing soil, 11 May 2007, Yogesh Joshi \& Santosh Joshi 07-008807 (LWG).

Notes - The species was reported from Jammu \& Kashmir by Zhurbenko (2013) and in the present study; it is extending its distributional range up to Uttarakhand.

ROSELLINULA R. Sant., Systema Ascomycetum 5: 311 (1986)

R. frustulosae (Vouaux) R. Sant., Thunbergia 6: 7, no. 119 (1988)

Host - Lecanora frustulosa 
Known distribution - Jammu \& Kashmir.

Notes - The species was reported from Jammu \& Kashmir by Zhurbenko (2013).

RHYMBOCARPUS Zopf, Hedwigia 35: 357 (1896)

*R. pertusariae Diederich, Zhurb. \& Etayo, The Lichenologist 32 (5): 468 (2000)

Hosts - Pertusaria coronata, P. leioplacella

Known distribution - Uttarakhand.

Materials examined - India, Uttarakhand, Jim Corbett National Park, Jhirna Srot, on thallus of P. leioplacella colonizing exposed Syzygium cuminii bark, 07 Nov 1997, D.K. Upreti L69860 (LWG 19439); Uttarakhand, Pithoragarh district, Sandev Botanical Hot spot, Deochula, alt. 2000$2318 \mathrm{~m}$, on thallus of $P$. coronata colonizing exposed bark, 27 Sep 2002, Vikas Pant 02000490/A (LWG 19595).

SARCOGYNE Flot., Bot. Ztg.: 753, 759 (1851)

S. lapponica (Ach. ex Schaer.) K. Knudsen \& Kocourk., Mycotaxon 105: 160 (2008)

Host - Acarospora sp., Carbonea sp.

Known distribution - Jammu \& Kashmir.

Notes - This species was reported from India by Awasthi (1991) under the name Polysporina dubia (H. Magn.) Vězda, and since then it was not reported from India.

S. sphaerospora J. Steiner, Österreichische Botanische Zeitschrift 49 (7): 251 (1899)

Host - Candelariella sp.

Known distribution - Jammu \& Kashmir.

Notes - The species was reported from Jammu \& Kashmir by Zhurbenko (2013).

SCLEROCOCCUM Fr., Trattato di knologia Ed. Sansoni Firenze: 79 (1819)

*S. simplex D. Hawksw., Bulletin of the British Museum for Natural History 6 (3): 249 (1979)

Host - Ochrolechia yasudae var. corallina, Pertusaria multipunctata

Known distribution - Sikkim, Uttarakhand.

Materials examined - India, Sikkim, South Sikkim district, Kupup, Gnathang (HSP-1), alt. 3903 m, on P. multipunctata colonizing twigs, 23 Apr 2014, R. Bajpai 14-024917 (LWG 29103). Uttarakhand, Bageshwar district, en route to Pindari, between Loharkhet and Dwali, alt. 1700-3000 m, on O. yasudae var. corallina colonizing Rhododendron bark, 21 May 1997, D.K. Upreti \& S. Chatterjee L68941 (LWG 13520).

*S. sphaerale (Ach.) Fr., Scleromyceti suecici no 179: no. 179 (1821)

Host - Pertusaria leucostoma

Known distribution - Madhya Pradesh.

Materials examined - India, Madhya Pradesh, Katni district, Piproel, $15 \mathrm{~km}$ away from Katni, on Pertusaria leucostoma colonizing Mangifera indica bark, 21 Feb 2009, G.K. Mishra \& S. Mohabe 09-009951/A (LWG 10568); 09-009952 (LWG 10561); 09-009954 (LWG 10529); 09009955 (LWG 10532).

SKYTTEA Sherwood, D. Hawksw. \& Coppins, Transactions of the British Mycological Society 75 (3): 482 (1981)

S. fusispora Sherwood, D. Hawksw. \& Coppins, Transactions of the British Mycological Society 75 (3): 484 (1981)

Host - Ochrolechia trochophora 
Known distribution - Assam.

Notes - The species was reported from Assam by Sherwood et al. (1981).

SPHAERELLOTHECIUM Zopf, Nova Acta Academiae Caesareae Leopoldino-Carolinae Germanicae Naturae Curiosorum 70: 184 (1897)

*S. atryneae (Arnold) Cl. Roux \& Triebel, Bulletin de la Société Linnéenne de Provence 45: 525 (1994)

Host - Protoparmeliopsis muralis

Known distribution - Uttarakhand.

Material examined - India, Uttarakhand, Chamoli district, on P. muralis colonizing exposed rock, 24 Sept 2006, S. Rawat 06-006779 (LWG).

*S. contextum Triebel, Bibliotheca Lichenologica 35: 76 (1989)

Host - Rhizoplaca chrysoleuca

Known distribution - Uttarakhand.

Materials examined - India, Uttarakhand, Bageshwar district, near Zero miles, Mirtoli to ridge of moraine, alt. $3600 \mathrm{~m}$, on $R$. chrysoleuca colonizing rocks, 11 Jun 1970, D.D. Awasthi 7713 (LWG); Chamoli district, Badrinath, east of temple, on way to Dhantoli village, alt. ca. $3250 \mathrm{~m}$, on $R$. chrysoleuca colonizing boulders, 29 Sept 1976, K. Dange 76.847 (LWG-LWU); Uttarkashi district, on way to Gomukh, $1 \mathrm{~km}$ from Gangotri, alt. $3230 \mathrm{~m}$, on $R$. chrysoleuca colonizing rocks, 30 Jun 1976, D.D. Awasthi \& S.R. Singh 8229 (LWG-AWAS 14909); Gomukh area, right bank, $3^{\text {rd }}$ and $4^{\text {th }}$ moraine, alt. ca. $3870 \mathrm{~m}$, on $R$. chrysoleuca colonizing boulders, 03 Jul 1976, D.D. Awasthi \& S.R. Singh 8449 (LWG-AWAS 14908); Gomukh area, right bank, $5^{\text {th }}$ moraine, alt. 3840 m, on $R$. chrysoleuca colonizing boulders, 04 Jul 1976, D.D. Awasthi \& S.R. Singh 8475 A (LWGAWAS 14915); Gomukh area, right bank, $5^{\text {th }}$ moraine, alt. $3840 \mathrm{~m}$, on $R$. chrysoleuca colonizing boulders, 04 Jul 1976, D.D. Awasthi \& S.R. Singh 8475 C (LWG-AWAS 14911).

*S. propinquellum (Nyl.) Cl. Roux \& Triebel, Bulletin de la Société Linnéenne de Provence 45: 530 (1994)

Host - Protoparmeliopsis muralis

Known distribution - Jammu \& Kashmir.

Material examined - India, Jammu \& Kashmir, Awantipur, 08 miles from Srinagar, on $P$. muralis colonizing rocks, 15 May 1962, R. Ghosh 97543 (LWG 15849).

*S. reticulatum (Zopf) Etayo, Cryptogamie Mycologie 29 (1): 87 (2008)

Host - Parmotrema reticulatum

Known distribution - Uttarakhand.

Materials examined - India, Uttarakhand, Almora district, en route to Daniya, Dhauladevi forest, on thallus of $P$. reticulatum, 16 May 2015, Krishna Chandra \& Shashi Upadhyay s.n. (KU); Binsar Wildlife Sanctuary, on thallus of $P$. reticulatum, 09 Aug 2015, Y. Joshi s.n. (KU); Nainital District, on way to Mukteshwar, alt. $2155 \mathrm{~m}$, on thallus of $P$. reticulatum colonizing rocks, $17 \mathrm{Jul}$ 2015, Shashi Upadhyay, P. Joshi, P. Chilwal, N. Kumar \& V. Kumar, s.n. (KU).

SPHAEROPEZIA Sacc., Botanisches Centralblatt 18: 253 (1884)

S. cf. lecanorae (Diederich \& G. Marson) Baloch \& Wedin, Mycologia 105 (2): 393 (2013) Host - Protoparmeliopsis muralis Known distribution - Jammu \& Kashmir. Notes - The species was reported from Jammu \& Kashmir by Zhurbenko (2013).

SPHINCTRINA Fr., Systema Orbis Vegetabilis 1: 120 (1825) 
S. anglica Nyl., Synopsis Methodica Lichenum Omnium hucusque Cognitorum, Praemissa Introductione Lingua Gallica 1: 143 (1860)

Host - Pertusaria sp.

Known distribution - Tamil Nadu, Uttarakhand.

Notes - The species was reported from India by Awasthi \& Singh (1975) and Pant \& Awasthi (1989).

S. tubaeformis A. Massal., Memorie Lichenographiche: 155 (1853)

Host - Pertusaria sp.

Known distribution - Assam, Manipur, Tamil Nadu, Uttarakhand.

Materials examined - India, Uttarakhand, Almora district, Jhakar Saim Forest, on thallus of Pertusaria sp. colonizing Quercus tree, 06 Jul 2014, M. Tripathi, K. Chandra \& S. Upadhyay s.n. (KU); Syahi Devi Forest, on thallus of Pertusaria sp. colonizing Quercus tree, 27 Jul 2014, S. Upadhyay \& N. Rana s.n. (KU).

Notes - The species was reported from Assam, Manipur, Tamil Nadu by Pant \& Awasthi (1989), while Joshi et al. (2015a) reported it from Uttarakhand.

SPIROGRAPHA Zahlbr., Nat. Pflanzenfamilien: 96 (1903)

*S. fusisporella (Nyl.) Zahlbr., Catalogus Lichenum Universalis: 96 (1903)

Host - Protoparmeliopsis muralis

Known distribution - Jammu \& Kashmir.

Material examined - India, Jammu \& Kashmir, Anantnag district, Baltal, alt. \pm 2745 m, on P. muralis colonizing rocks, 29 Aug 1982, D.K. Upreti 13923 (LWG 15851).

STIGMIDIUM Trevis., Conspectus Verrucarinarum. Prospetto dei Generi e delle Specie de Licheni Verrucarini: 17 (1860)

*S. cerinae Cl. Roux \& Triebel, Bulletin de la Société Linnéenne de Provence 45: 480 (1994)

Host - Xanthoria elegans

Known distribution - Uttarakhand.

Material examined - India, Uttarakhand, Chamoli district, Malari, on X. elegans colonizing rocks, 24 Sept 2006, S. Rawat 06-006778 (LWG)

*S. frigidum (Sacc.) Alstrup \& D. Hawksw., Meddelelser om Grønland Biosciences 31: 67 (1990)

Host - Thamnolia vermicularis

Known distribution - Arunachal Pradesh.

Material examined - India, Arunachal Pradesh, Tawang district, Nagula, alt. 4290 m, on T. vermicularis growing on soil, 25 Sept 2012, Rupam Debnolti 12-017715 (LWG)

S. gyrophorarum (Arnold) D. Hawksw., Kew Bulletin 30 (1): 201 (1975)

Host - Umbilicaria vellea

Known distribution - Jammu \& Kashmir.

Notes - The species was reported from Jammu \& Kashmir by Zhurbenko (2013).

S. pumilum (Lettau) Matzer \& Hafellner, Bibliotheca Lichenologica 37: 115 (1990)

Hosts - Phaeophyscia ciliata, P. primaria, Physcia caesia, Physcia sp.

Known distribution - Jammu \& Kashmir, Sikkim, Uttarakhand.

Materials examined - India, Jammu \& Kashmir, Pahalgam, on way to Chandanwari, alt. ca.

$2700 \mathrm{~m}$, on P. caesia colonizing rocks, 29 Jun 1977, K. Dange 77.312 (LWG-LWU). Sikkim,

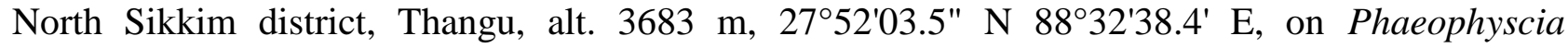


primaria colonizing rocks over mosses, 27 Apr 2014, R. Bajpai 14-025004 (LWG 29740). Uttarakhand, Bageshwar district, near Pindari glacier, Mirtoli to ridge moraine, alt. $3660 \mathrm{~m}$, on Physcia caesia colonizing on moss over stones, 11 Jun 1970, D.D. Awasthi 7702 (LWG-AWAS 16149); Chamoli district, Kedarnath hill side, on west of the temple, alt. 3580-3625 m, on P. caesia colonizing rocks, 19 Sept 1976, K. Dange 76-197 (LWG-LWU 05267); Tehri Garhwal, above Jamunotri, alt. $3960 \mathrm{~m}$, on thallus of Physcia sp. growing along with Protoparmeliopsis muralis colonizing rocks, 22 Jun 1951, D.D. Awasthi 908 (LWG-AWAS 13412).

Notes - The species was previously reported by Zhurbenko (2013) from Jammu \& Kashmir, colonizing Phaeophyscia ciliata. In the present study, the species is extending its distibutional range as well as host range.

S. tabacinae (Arnold) Triebel, Bibliotheca Lichenologica 35: 236 (1989)

Host - Toninia tristis ssp. asiae-centralis

Known distribution - Jammu \& Kashmir.

Material examined - India, Jammu \& Kashmir, Leh district, Yeru, alt. 3600 m, on T. tristis ssp. asiae-centralis colonizing soil among mosses, 06 Aug 2003, D.K. Upreti \& S. Chatterjee 03001792/B (LWG).

*S. xanthoparmeliarum Hafellner, Bulletin de la Société Linnéenne de Provence 44: 231 (1994)

Host - Xanthoparmelia stenophylla

Known distribution - Jammu \& Kashmir.

Material examined - India, Jammu \& Kashmir, Kashmir, on X. stenophylla colonizing bark, 1970, s.n. (LWG).

TAENIOLELLA S. Hughes, Canadian Journal of Botany 36 (6): 816 (1958)

*T. delicata M.S. Christ. \& D. Hawksw., Bulletin of the British Museum for Natural History 6 (3): 253 (1979)

Host - Heterodermia sp.

Known distribution - Uttarakhand.

Material examined - India, Uttarakhand, Nainital District, on way to Mukteshwar, alt. 2155 m, on thallus of Heterodermia sp., 17 Jul 2015, Shashi Upadhyay, P. Joshi, P. Chilwal, N. Kumar \& V. Kumar s.n. (KU).

VOUAUXIELLA Petr. \& Syd., Feddes Repertorium Specierum Novarum Regni Vegetabilis Beiheft 42: 482 (1927)

V. lichenicola (Linds.) Petr. \& Syd., Feddes Repertorium Specierum Novarum Regni Vegetabilis Beiheft 42: 484 (1927)

Host - Lecanora cf. subrugosa

Known distribution - Jammu \& Kashmir.

Material examined - India, Jammu \& Kashmir, Baramullah district, Gulmarg, east side, alt. $2560 \mathrm{~m}$, on L. cf. subrugosa colonizing trunk of Cedrus deodara, 25 Jul 2005, M. Sheikh 05006059 (LWG 15489).

ZWACKHIOMYCES Grube \& Hafellner, Nova Hedwigia 51 (3-4): 305 (1990)

Z. coepulonus (Norman) Grube \& R. Sant., Nova Hedwigia 51 (3-4): 310 (1990)

Host - Xanthoria elegans

Known distribution - Jammu \& Kashmir.

Notes - The species was reported from Jammu \& Kashmir by Zhurbenko (2013). 
Z. cf. kiszkianus D. Hawksw. \& Miadl., Mycological Research 101 (9): 1131 (1997)

Host - Peltigera elisabethae

Known distribution - Jammu \& Kashmir.

Notes - The species was reported from Jammu \& Kashmir by Zhurbenko (2013).

*Z. lecanorae (Stein) Nik. Hoffm. \& Hafellner, Bibliotheca Lichenologica 77: 124 (2000)

Host - Lecanora sp.

Known distribution - Jammu \& Kashmir.

Material examined - India, Jammu \& Kashmir, Ladakh, Zansbar, Zangla, on Lecanora growing along with Candelariella grimmiae colonizing rocks, 06 Sept 2012, Jatinder Kumar 12019228 (LWG 13384).

\section{Excluded taxon}

DENDRISCOCAULON Nyl., Lichenes Novae Zelandiae: 11 (1888)

D. umhausense (Auersw.) Degel., Arkiv før Botanik 30A: 28 (1942)

Notes - It was reported from Kerala and Tamil Nadu by Awasthi (1965, 1991), but is excluded from the study, since it is not a lichenicolous fungus.

\section{Discussion}

The revision of lichen collections from India yielded 105 species of lichenicolous fungi from 53 genera and 02 species of lichens from 02 genera optionally or permanently growing on other lichens, which makes India one of the best-studied areas in South East Asia regarding lichenicolous mycobiota. Thus, the revealed species richness of the lichenicolous mycota in India increased from 52 to 105 . However, this number is very small, many groups of lichens are still not fully explored out in the present study; hence, the actual number of lichenicolous fungi species for the country will increase a lot in the future.

The following eight lichen genera are new hosts for nine species of lichenicolous fungi: Candelariella for Endococcus perpusillus; Lobaria for Nectriopsis lecanodes; Ochrolechia for Buelliella minimula, Sclerococcum simplex; Pertusaria for Cladosporium lichenophilum; Rhizocarpon for Lichenodiplis lecanorae, Lichenostigma triseptatum; Psora for Muellerella lichenicola and Physcia for Stigmidium pumilum. Besides this, the lichen Monerolechia badia is for the first time documented on Acarospora. These data must be considered with some hesitation because many species of lichenicolous are very much dependent on the host.

There is a very insufficient knowledge of lichenicolous biota in India; hence, not much data is available on the frequency of occurrence of lichenicolous fungi in nature till many more collections are available. This fact can be justified by the high percentage of species $(30 \%)$ that are known from a single finding. So far, 38 species are represented by five or more than five collections, viz. Arthonia clemens (6), A. molendoi (18), Cercidospora caudata (7), C.macrospora (14), C. melanophthalmae (18), Cercidospora sp. (11), C. xanthoriae (14), Cladosporium licheniphilum (8), Corticifraga peltigerae (10), Dactylospora saxatilis (5), Endococcus rugulosus (8), Intralichen christiansenii (14), Kalchbrenneriella cyanescens (11), Lichenoconium lecanorae (5), L. xanthoriae (08), Lichenodiplis lecanorae (14), Lichenostigma alpinum (7), L. cosmopolites (38), L. cf. elongatum (11), Marchandiomyces corallinus (7), Monerolechia badia (10), Monodictys epilepraria (6), Muellerella erratica (32), M. lichenicola (5), M. pygmaea (10), Nectriopsis lecanodes (7), Opegrapha foreaui (10), Phoma sp. (6), Polycoccum clauzadei (13), P. pulvinatum (7), Pyrenidium actinellum (9), Rosellinula frustulosae (13), Sarcogyne sphaerospora (10), Sphaerellothecium contextum (6), Sphaeropezia cf. lecanorae (6), Stigmidium pumilum (5), S. tabacinae (10) and Zwackhiomyces coepulonus (11), while 33 of the 105 discovered species are represented by a single finding. 
The most common species of lichenicolous biota in the study area seem to be Cercidospora macrospora, C. melanophthalmae, Cercidospora sp., C. xanthoriae, Corticifraga peltigerae, Intralichen christiansenii, Lichenodiplis lecanorae, Lichenostigma cosmopolites, L. cf. elongatum, Monerolechia badia, Muellerella erratica, M. pygmaea, Polycoccum clauzadei, Rosellinula frustulosae, Sarcogyne sphaerospora, Stigmidium tabacinae and Zwackhiomyces coepulonus.

The study on lichenicolous biota of India is in its preliminary stage, hence, if we consider Lichenicolous Index (the ratio of species of lichenicolous fungi per species of lichens within an area) of the Holarctic (1:5 Zhurbenko 2007) or Japan (1:3 Zhurbenko et al. 2015) applicable to India, then the expected diversity of lichenicolous fungi in India would be about 450-800 species. Despite long history of lichenological studies in India, until now only four species of lichenicolous fungi were described as new to science by early authors (Moreau 1951a,b; Sherwood et al. 1981; Hariharan et al. 1996; Coppins \& Kondratyuk 1998; Joshi et al. 2013), and 48 species of lichenicolous fungi were newly reported from the county (Joshi 2015a,b; Zhurbenko 2013). The percentage of taxonomic novelties in the examined material may even be higher as additional new taxa are expected to be hidden among the tentatively named Cercidospora sp., Lichenostigma cf. elongatum, Lichenostigma subgen. Lichenogramma sp., Phoma sp., Sphaeropezia cf. lecanorae and Zwackhiomyces cf. kiszkianus, and many more lichens remained to be searched out for lichenicolous biota.

Besides lichenicolous biota, three species of lichens viz. Peltigera ponojensis, P. scabrosa and Seirophora contortuplicata, which are quite common and widely distributed in mountain regions of the northern hemisphere and recently reported from Jammu \& Kashmir (Zhurbenko 2013), were not only missing in Compendium of the macrolichens of India (Awasthi 2007), but also in Annotated checklist of Indian lichens (Singh \& Sinha 2010), thus, needs to be added to any upcoming checklist on Indian lichen biota.

\section{Acknowledgements}

One of the authors (YJ) would like to thank Scientific and Engineering Research Board [SB/FT/LS-313/2012] and University Grants Commission [41-488/2012 (SR)] for financial assistance, and to Drs. Paul Diederich, Uwe Braun, M. Zhurbeko, J. Etayo and David Hawksworth for their generous help regarding the identification of some samples and providing valuable literature. YJ would also like to acknowledge Prof. Anil Joshi for linguistic corrections and to all the collectors who have collected lichen samples. The authors are indebted to Director, Kerala Forest Research Institute, Peechi, Kerala; Director, CSIR-National Botanical Research Institute and Head, Department of Botany, S.S.J. Campus, Almora for providing laboratory facilities.

\section{References}

Alstrup V, Ahti T. 2007 - New reports of lichenicolous fungi, mainly from Finland and Russia. Karstenia 47, 1-4.

Awasthi DD, Singh KP. 1975 - Additions to the lichen flora of India - III. Geophytology 5, 110112.

Awasthi DD. 1965 - Catalogue of the lichens from India, Nepal, Pakistan, and Ceylon. Weinheim, Verlag von J. Cramer.

Awasthi DD. 1991 - A key to microlichens of India, Nepal and Srilanka. Bibliotheca Lichenologica 40, 1-337.

Awasthi DD. 2007 - A compendium of the macrolichens from India, Nepal and Sri Lanka. Bishen Singh Mahendra Pal Singh, Dehra Dun, India.

Coppins BJ, Kondratyuk SY. 1998 - Opegrapha trassii sp. nov., a new lichenicolous fungus on Heterodermia. Folia Cryptogamica Estonica 32, 9-14.

Hariharan GN, Mibey RK, Hawksworth DL. 1996 - A new species of Lichenopeltella on Porina in India. Lichenologist 28, 294-296. 
Hertel, H. 1983 - Über einige aus Lecidea und Melanolecia (Ascomycetes, Lichenisati) auszuschliessende Arten. Mitteilungen der Botanischen Staatssammlung München 19, 441447.

Joseph S, Sinha GP. 2015 - The lichenicolous species of Melaspilea (Melaspileaceae) in India. Taiwania 60(1), 18-22.

Joshi S, Upreti DK, Nayaka S. 2013 - A new lichenicolous Arthonia species (Arthoniaceae) on Diorygma from India. Lichenologist 45, 323-327.

Joshi Y, Upadhyay S, Shukla S, Nayaka S, Rawal RS. 2015a - New records and an updated checklist of lichenicolous fungi from India. Mycosphere 6(2), 195-200.

Joshi Y, Upadhyay S, Tripathi M, Chandra K. 2015b - First report of a lichenicolous fungus from India Opegrapha phaeophysciae. Kavaka 44, 50-52.

Moreau F. 1951a - Sur une Dothidéacée parasite du Lihen Anaptychia speciosa (Wulfen) Massalongo. Bulletin of the Botanical Society France 98: 7-9.

Moreau F. 1951b - Un champignon lichenicole cecidogene, Phyllosticta galligena, sp. nov. Bulletin of the Botanical Society France 98, 100-102.

Pant G, Awasthi DD. 1989 - Caliciales from India and Nepal. Biovigyanam 15, 3-27.

Poelt J. 1961 - Flechten aus dem N.W. Karakorum in Rahmen den Duetschen Karakorum expedition 1959, von F. Lobbichler und Dr. Schneider gessammelt. Mitteilungen der Botanischen Staatssammlung München 4, 83-94.

Sherwood MA, Hawksworth DL, Coppins BJ. 1981 - Skyttea, a new genus of odontotremoid lichenicolous fungi. Transactions of the British Mycological Society 75, 479-490.

Singh KP, Sinha GP. 2010 - Indian Lichens: An Annotated Checklist. Botanical Survey of India, Kolkata, India.

Triebel D. 1989 - Lecideicole Ascomyceten. Eine Revision der obligat lichenicolen Ascomyceten auflecideoiden Flechten. Bibliotheca Lichenologica 35, 1-278.

Zhurbenko MP, Frisch A, Ohmura Y, Thor G. 2015 - Lichenicolous fungi from Japan and Korea: new species, new records and a first synopsis for Japan. Herzogia 28, 762-789.

Zhurbenko MP. 2007 - Lichenicolous fungi of Russia: history and first synthesis of exploration. Mikologiya I Fitopatologiya 41, 481-486.

Zhurbenko MP. 2013 - A first list of lichenicolous fungi from India. Mycobiota 3, 19-34. 